\title{
Paysage urbain et identités collectives à Kaliningrad
}

\author{
Colin HORENBEEK \\ Chercheur indépendant \\ Auteur d'un mémoire de Master sur le sujet \\ Université libre de Bruxelles (BE) \\ colin@horenbeek.be
}

\begin{abstract}
Résumé
Le paysage urbain est un révélateur de l'organisation d'une société ainsi que des stratégies politiques et sociales qui s'y développent. Kaliningrad n'est à ce titre pas en reste. La ville russe, dont la région éponyme est enclavée au sein de l'Union européenne, a connu de nombreuses ruptures historiques que l'on retrouve dans son aménagement. Königsberg, ville acquise par l'Union soviétique en 1946 après la fin de la Seconde Guerre mondiale, est renommée Kaliningrad et doit modéliser sa nouvelle identité soviétique. Le passé allemand de la ville est effacé des narrations officielles. Véritable trophée de guerre, l'aménagement de la ville structure l'organisation de la vie sociale et célèbre la victoire soviétique par de nombreux monuments. À la fin du XXe siècle, Kaliningrad retrouve Königsberg, la ville allemande dont il ne reste que peu de traces matérielles. L'ouverture historiographique s'accompagne d'un processus de rematérialisation de l'identité allemande dans le paysage urbain, qui devient un nouvel attribut identitaire pour la ville autant qu'une attraction touristique dissonante. Cette période est synonyme d'autonomie importante pour les autorités locales à la suite de la chute de l'URSS, avant d'être interrompue au début des années 2000 par le renforcement de la verticale du pouvoir fédéral et l'affirmation nationale russe dans les espaces urbains à Kaliningrad. Les débats récurrents pour renommer la ville n’aboutiront pas. À travers ces ruptures, la population locale se ménage un espace identitaire propre, sans se distancier pour autant de l'appartenance russe. Un phénomène qu'illustre particulièrement l'appellation locale de la ville actuelle par ses jeunes habitants : « Kenig », forme raccourcie de Königsberg.
\end{abstract}

Mots-clés : paysage urbain, Kaliningrad, Königsberg, identité collective.

\begin{abstract}
Urban landscape is an indicator which reveals the organisation of society as well as the political and social strategies it develops. Kaliningrad is not new to this. The Russian city and region of the same name, landlocked within the European Union, has experienced many historical ruptures which can be seen in its landscape. Kaliningrad, the Soviet city, acquired and renamed in 1946 after the end of the Second World War, had to model its new Soviet identity. The city's German past was erased from official narratives. As a war trophy, the city's layout structures the organisation of social life and celebrates the Soviet victory with numerous monuments. At the end of the 20th century, Kaliningrad rediscovers Königsberg, the German city of which few material traces remain. This historiographical opening is accompanied by a new materialisation process of the German identity in the urban landscape, which becomes a new identity attribute for the city as well as a dissonant tourist attraction. This period is synonymous with significant autonomy for local authorities following the fall of the USSR, interrupted at the beginning of the 2000s by the strengthening of the vertical of federal power and the Russian national affirmation in urban areas in Kaliningrad. Recurrent debates to rename the city will not succeed. Regardless of these ruptures, the local population created its own identity, without completely distancing itself from its Russian belonging. A phenomenon that is particularly illustrated by the local name of the current city used by its young inhabitants: "Kenig," a shortened form for Königsberg.
\end{abstract}

Keywords: urban landscape, Kaliningrad, Königsberg, collective identity. 
Introduction

1. Kaliningrad : l’identité soviétique et ses symboles

1.1 La matérialité du trophée de guerre

1.2 L'esprit de conquête soviétique

123

1.3 Une architecture de la surveillance

2. « Königsberg » : rematérialiser l'identité allemande

2.1 Reconstruction d'un héritage culturel

126

2.2 Königsberg comme parc thématique

127

3. Entre « Kantgrad » et « Severnaja Rus" » : matérialiser une appartenance commune post-soviétique

3.1 La réorganisation de l'héritage soviétique

130

3.2 Les « nouveaux » héros

131

3.3 L'étranger au service du national

132

4. « Kenig » : vers l'émergence d'une identité locale dissonante?

4.1 « Kenig» : compromis ou aliénation? $\quad 134$

4.2 De l'appropriation à la mobilisation $\quad 135$

$\begin{array}{ll}\text { Conclusion } & 137\end{array}$

Références bibliographiques $\quad 138$

Annexe : vignettes illustratives $\quad 140-144$

Illustrations $\quad 145-154$ 


\title{
Paysage urbain et identités collectives à Kaliningrad
}

\author{
Introduction
}

\begin{abstract}
« Trophée de guerre comme les îles Kouriles soustraites au Japon, Kaliningrad était devenue russe, cela sautait aux yeux. Dévêtue d'un coup, revêtue aussitôt.

Qui aurait pu résister à la violence d'une telle mise à nu et à la brutalité de ce nouvel accoutrement? Cependant, en examinant de plus près les rues, les maisons, les parcs, le suintement du passé allemand remontait partout à la surface. Comment stopper cette hémorragie d'une époque honnie ? L'architecture néo-médiévale et néobaroque entrapparaissait. Les fortifications prussiennes revêtues de briques rouges étaient intactes ». (Kauffmann 2016, 22)
\end{abstract}

Le paysage urbain véhicule des représentations des identités collectives des populations qui l'habitent. La ville de Kaliningrad actuelle laisse entrevoir plusieurs couches historiques de ce processus de représentation qui sont à la fois complémentaires et antagonistes. Plusieurs villes dans la ville. En 1946, lorsque le territoire de Königsberg est négocié par Staline à la conférence d'après-guerre de Potsdam, la ville est en ruines. Une grande partie du bâti a été rasée par les bombardements alliés (Diener et Hagen 2011). La ville qui naît sur ce territoire au moment de sa reconstruction, c'est Kaliningrad, symbole de la nouvelle identité soviétique de la région. Cette ville est habitée par une population transmigrée depuis différentes régions d'URSS (principalement la RSFSR) ainsi que par des soldats soviétiques rapatriés d'Allemagne de l'Est. Le passé allemand de Kaliningrad, représentant aux yeux des autorités le « militarisme impérialiste fasciste », doit être oublié (Eaton 2013).

Plusieurs décennies plus tard, après la tentative d'ouverture de la mémoire soviétique par Mikhaïl Gorbatchev et sa glasnost dans les années 1980, ce passé est réhabilité et les habitants de la ville, dont on avait jusqu'alors effacé l'histoire, peuvent se réapproprier la ville de Königsberg. Cette ville prussienne, malgré le peu de structures d'époque qui ont subsisté, réapparaîtra dans les espaces urbains dès les années 1990 et formera, à mesure qu'elle se fait publique et visible, une nouvelle page de la composante territoriale de l'identité collective de la ville et ses habitants.

En 1991, la chute de l'Union soviétique et l'émergence de la Fédération de Russie imposent à ce territoire une nouvelle rupture. La première décennie post-soviétique est synonyme d'autonomie accrue pour les pouvoirs locaux, 
tournés vers leurs voisins directs et l'Europe occidentale. Une période également caractérisée par la désorganisation et l'idiosyncrasie. Un tournant dans l'affirmation d'une identité locale spécifique, entre Kaliningrad et Königsberg, entre Russie et Union européenne. Avec l'élection de Vladimir Poutine à la présidence en 2000, la réaffirmation de l'identité russe des marges du territoire se fait croissante, à mesure que la verticale du pouvoir se renforce. À leur tour, les autorités fédérales mobilisent leurs propres symboles et tentent de construire un sentiment d'appartenance nationale en capitalisant sur certains éléments identitaires nouveaux ou réinvestis. Cette nouvelle couche de l'aménagement de la ville génère de nouveaux référents identitaires, une troisième ville dans la ville en à peine un demi-siècle. Plus encore, depuis 2010 et l'éclatement de protestations locales, les autorités de la ville de Kaliningrad sont sous surveillance. Le maire actuel, Aleksej Silanov, a été coopté par le gouverneur régional Anton Alihanov (Хлебников 2018). Ce dernier est en poste depuis 2017, suite à sa nomination par Vladimir Poutine et aux élections régionales marquées par d'importantes irrégularités (Голос 2017), assurant une verticale du pouvoir du fédéral vers le local, par l'intermédiaire de la région. Autant de manœuvres politiques pourtant loin d'effacer les questionnements identitaires locaux (Kowalczyk et al. 2019).

Quelles sont les conséquences de cette multiplicité d'histoires, d'espaces et d'identités dans un même ensemble urbain ? Au regard des nombreuses ruptures géographiques qu'a vécues Kaliningrad, jinterroge au cours de cet article l'impact de la matérialisation de ces différentes époques sur le processus de construction identitaire que connaissent la ville et ses habitants. Qu'est-ce qui se cache derrière le terme " Kenig », utilisé aujourd'hui par un grand nombre de jeunes habitants pour désigner leur ville ? Cette forme raccourcie de Königsberg signifie-t-elle un déni de Kaliningrad ? Un retour en force de Königsberg et du passé allemand ? Ou peut-être cela signifie-t-il que les différentes identités territoriales en présence se confrontent et se fusionnent, pour générer une identité locale spécifique et peutêtre, dissonante? (voir Figure 1) 


\section{Kaliningrad : l'identité soviétique et ses symboles}

"Les monuments devraient être érigés en l'honneur des personnages remarquables dans les domaines des activités révolutionnaires et sociales, de la philosophie, la littérature, les sciences et les arts. Les monuments devraient être

érigés sur les boulevards, dans les jardins publics et les espaces similaires dans tous les quartiers de Moscou, avec des citations ou des maximes gravées sur leurs piédestaux ou leurs contours, pour que ces monuments puissent apparaître comme des tribunes de rue desquelles les mots voleraient vers les masses populaires, stimulant les esprits et les consciences ${ }^{1}$.

En 1945, les troupes soviétiques envahissent Königsberg. Simple étape sur la route des Soviétiques vers Berlin? Pas vraiment. Ce territoire occupe une position géostratégique dont Joseph Staline a bien conscience. Lors du processus de négociation de la fin de la Seconde Guerre mondiale, ce dernier place ses cartes et avance une ambition qui va s'avérer payante : s'approprier la PrusseOrientale. Outre l'accès à la mer Baltique libre de glaces toute l'année et la consolidation de l'assise soviétique sur la région baltique après l'annexion des trois républiques baltes, ce territoire porte une symbolique sur mesure. C'est le trophée soviétique qui célèbre la victoire de l'URSS pendant la « Grande Guerre patriotique » [Velikaja Otečestvennaja vojna], mettant en avant la bravoure des soldats et la puissance du projet communiste, face au « militarisme impérialiste fasciste » dont Königsberg était, selon Staline, la représentation parfaite (Diener et Hagen 2011) ${ }^{2}$.

La réorganisation identitaire et infrastructurelle de la région est précédée par une première phase d'archéologie politique. Tous les moyens sont mis en place pour prouver « scientifiquement » les racines slaves de ce territoire, permettant d'en légitimer l'appropriation. Aucun historien ne pourra néanmoins prouver les liens du territoire avec une prétendue « civilisation slave » (Sezneva 2013 ; Dementyev 2015). En parallèle, le territoire est repeuplé de Russes et de quelques autres communautés ethniques soviétiques - Biélorusses, Ukrainiens et Lituaniens essentiellement - (Kolossov et Radvanyi 2014). Ces populations sont déplacées

1 Traduction de l'auteur depuis l'anglais. Mémorandum du Collège artistique du Commissariat de l'Éducation de Moscou au Conseil des commissaires du peuple, sur l'édification à Moscou de 50 monuments en l'honneur de personnages remarquables de la sphère des activités sociales et révolutionnaires, en philosophie, littérature, sciences et arts. La date exacte du texte, le 18 juin 1918, apparaît dans une publication postérieure au « Mémorandum ». (Б. Н. Терновец, « 15 лет советской скульптуры », Искусство 3 (1933) : 152-153, cité dans Larissa Alekseevna Zhadova (eds), Tatlin, (Thames and Hudson, 1988),185 (porté à ma connaissance au cours de l'entretien avec Jamie Freeman, via vidéoconférence depuis Bruxelles, le 1 juillet 2019).

2 Néanmoins, la Prusse-Orientale est quelques peu redécoupée, de sorte que l'actuel territoire de Kaliningrad est plus petit que celui originellement occupé par la Prusse-Orientale. Plus de $50 \%$ du territoire est attribué à la Pologne en 1946. De même, une petite région au nord du fleuve Niémen, le district de Memel, est rattachée à la Lituanie dès 1918 (Tétart 2007). 
dans le contexte chaotique de l'après-guerre, alors que leurs régions natales ont été bien souvent détruites et leurs familles décimées. Elles viennent de plus d'une cinquantaine de régions différentes et sont relogées à Kaliningrad avec une grande promesse : celle d'une nouvelle chance, d'une nouvelle vie, et de nombreuses aides d'État financières et matérielles (Eaton 2013). La reconstruction de Kaliningrad, renommée d'après le nom du président du Soviet suprême Mihail Ivanovič Kalinin, peut commencer. Oubliez les hautes façades hanséatiques en bois ou en brique rouge et le bouillonnement culturel de Königsberg. Place au fonctionnalisme, au modernisme, au béton et aux grandes avenues, caractéristiques qui s'inscrivent dans le projet soviétique de réorganisation de la vie sociale par l'aménagement du territoire, autant que dans les critères esthétiques et sociaux de l'après-guerre sur tout le continent européen (Hirt 2013 ; Lebow 2013 ; Lenormand 2014).

\subsection{La matérialité du trophée de guerre}

À travers leurs gigantesques projets urbains, les autorités soviétiques ont dessiné à de nombreuses reprises les parcs comme des espaces de célébration de la victoire militaire, faisant des "parcs de la Victoire » [Park Pobedy] des éléments urbains essentiels dans de nombreuses villes soviétiques ${ }^{3}$. Si l'exemple le plus notable est probablement le parc de la Victoire de Moscou (Bouchard 2013), le parc de la Victoire de Kaliningrad, aménagé dès l'acquisition de la ville, n'est pas en reste ${ }^{4}$. À travers ses différents monuments, il représente la rupture historique et territoriale opérée en 1945 et matérialise le souvenir de l'héroïsme du peuple soviétique, victorieux « grâce à ses valeurs plus encore que par son armée » (Ibid.).

Les centaines de fleurs, déposées aux pieds de chacun des monuments du parc le 9 mai, jour de commémoration de la victoire en URSS et dans une partie de l'espace post-soviétique, semblent attester de la vivacité de ce souvenir (Ibid.) (voir Figure 2).

La région de Kaliningrad représente au sortir de la guerre un trophée, celui d'une victoire dont les autorités de l'époque souhaitent le souvenir pérenne et vivace. Le parc de la Victoire de Kaliningrad n'en est pas le seul témoin. De nombreux autres monuments sont installés dans les années qui suivent la fin de la Seconde Guerre mondiale. Ils rappellent la victoire soviétique et sont déclinés en différentes représentations monumentales de militaires, d'armements, de généraux ou de soldats, qui continuent encore aujourd'hui de faire vivre le

3 Entretien avec Jamie Freeman, historien de l'Université d'East Anglia, Grande-Bretagne, et auteur d'une thèse de doctorat sur les aménagements soviétiques de Kaliningrad, 1 juillet 2019, via vidéoconférence depuis Bruxelles.

4 Ibid.

CONNEXE 6 | 2020 - Espace baltique : dynamiques identitaires et stratégies politiques en question(s) 
souvenir de la «libération héroïque » de la ville. Ils pérennisent également une narration historique idéologisée, toujours matérielle à Kaliningrad malgré la chute de l'URSS. Ces aménagements permettent à un imaginaire militaire d'exister au cœur d'espaces pratiqués au quotidien par des générations qui, en 2020, n'ont parfois plus de membre de leur famille ayant vécu la réalité de ce conflit. Si les monuments de guerre permettent à la mémoire de s'inscrire dans la durée, ils peuvent également maintenir des représentations militaires dans la vie de tous les jours des individus, reflétant des identités que l'on pourrait qualifier de "post-conflit », non parce qu'elles marquent la fin de l'existence de tout conflit armé, mais parce qu'elles sont structurées par les représentations d'un conflit passé. Le «Monument aux Tankistes » de la Seconde Guerre mondiale [Pamjatnik « Gerojam-tankistam »] illustre cette observation. Installé au centre d'un îlot d'immeubles dans une zone résidentielle de la ville en 1980, il insère dans le paysage du quotidien une représentation militaire lourde de $\operatorname{sens}^{5}$ (voir Figure 3).

\subsection{L'esprit de conquête soviétique}

"L'humanité ne restera pas sur Terre pour toujours, mais, à la recherche de lumière et d'espace, elle pénétrera d'abord timidement les limites de l'atmosphère avant de finalement conquérir l'Espace et le système solaire $»^{6}$.

À proximité de la berge du «Musée de l'Océan Mondial »[Muzej Mirovogo okeana] de Kaliningrad, une longue stèle de marbre posée sur un muret en 1978 commémore la conquête de l'extrême nord de l'océan Atlantique par les marins soviétiques, en particulier une expédition menée en 1948. Plus au nord dans la ville, un impressionnant monument célèbre l'héroïsme des astronautes soviétiques (installé en 1980). Il fait face à une fresque, au cœur d'une rue commerçante. Cette dernière représente le visage d'un autre astronaute, Aleksej Leonov, particulièrement apprécié à Kaliningrad. Ces différentes images et figures des conquêtes soviétiques s'insèrent dans une narration sociale spécifique. La célébration des héros de la conquête spatiale comme maritime constituait un élément idéologique important en Union soviétique. Nourri par une grande soif de découverte et de progrès technique, le système soviétique s'est donné des moyens importants pour la recherche scientifique, militaire ou spatiale, autant que pour exploiter les confins de son propre territoire. Cette caractéristique a marqué de son empreinte le paysage urbain de Kaliningrad à

5 Les berges du « Musée de l'Océan Mondial » de Kaliningrad sont également illustratives. Elles constituent une forme de " brocante soviétique " faite de différentes reliques militaires : sous-marin, navire de l'agence Roskosmos, avion militaire ou encore mines sous-marines, issues de différentes époques.

6 Citation du scientifique soviétique Konstantin Ciolkovskij, inscrite sur le monument aux héros de la conquête de l'Espace à Kaliningrad. Observation de terrain, 15.04.2018, Kaliningrad. 
l'instar de son identité de trophée de guerre. À l'image de l'astronaute Aleksej Leonov, citoyen d'honneur de la ville, les représentations matérielles et personnifications des progrès techniques soviétiques structurent l'expérience du paysage urbain de Kaliningrad et caractérisent l'identité soviétique de la ville, empreinte d'un important imaginaire de conquête et de progrès (voir Figure 4).

\subsection{Une architecture de la surveillance}

Certains habitants l'appellent aujourd'hui « le monstre » [urod] (Berger 2010 ; Sezneva 2013 ; Amundsen 2019) ou « le robot » [robot] (Amundsen 2019). Après toutes ces années, sans jamais avoir été occupée ni utilisée, la Maison des Soviets [Dom Sovetov] est toujours debout. Visible de presque partout au centre-ville, elle domine le paysage urbain de Kaliningrad et constitue un lourd héritage. À travers sa position, son architecture, et l'institution qu'elle devait abriter, le Soviet suprême, elle représente à elle seule toute la rigidité de l'organisation étatique soviétique. Elle illustre la place symbolique de Kaliningrad, qui constituait dès 1946 un avant-poste de surveillance vers l'extérieur autant qu'un territoire sous surveillance. Initialement conçu par l'administration Brejnev en 1968 pour accueillir les fonctionnaires soviétiques à Kaliningrad, le bâtiment à l'architecture brutaliste connaîtra un tout autre sort. Si la structure extérieure du bâtiment est terminée (sa construction débute en 1980), ses aménagements intérieurs ne seront jamais entrepris et il ne sera jamais occupé. En cause, un problème de stabilité du sol, rendant possible un effondrement de la structure (Berger 2010). Les autorités soviétiques ont tracé dans la ville de Kaliningrad le sillon de leurs représentations de l'organisation politique de la société nouvelle. Cette société est marquée par un appareil d'État puissant et répressif, mais aussi particulièrement visible et représentée par des structures bâties imposantes. La Maison des Soviets [Dom Sovetov] fait, depuis 2018, l'objet d'un projet de réaménagement porté par le gouvernement régional et un investisseur privé. Néanmoins, en octobre 2020, les travaux n'avaient pas encore pu commencer. Le bâtiment est squatté et est l'objet de luttes entre les différents niveaux de pouvoir (NoviiKaliningrad.ru 2018a). Entre rapports de force et récupération politique, la Maison des Soviets semble vouée à rester le fantôme le plus visible de Kaliningrad (voir Figure 5). 
2. « Königsberg » : rematérialiser l'identité allemande

« Regardez ces magnifiques bâtiments ! À l'origine, c'était de bien tristes krouchtchëvki. Mais aujourd'hui, elles ont été transformées en magnifiques et vivantes bâtisses romantiques rappelant Königsberg $»^{7}$ !

L'histoire allemande et prussienne de la ville, rayée de la carte tant par les bombardements alliés que par le discours soviétique, sonne son retour à la chute de l'Union soviétique. Dans les années 1960, les premiers questionnements émergent quant à la réhabilitation de la mémoire allemande du territoire (Berger 2010 ; Serrier 2010 ; Diener and Hagen 2011). À la fin des années 1980, la glasnost de Mikhail Gorbatchev permet à ces velléités de commencer à s'exprimer (Berger 2010 ; Dementyev 2015). Mais c'est en 1991 que le processus de rematérialisation de l'identité allemande de la ville commence. Kaliningrad obtient le statut de "ville historique ». Ce statut n'ayant pas de conséquences légales et urbanistiques, il marque surtout la réhabilitation de l'histoire présoviétique du territoire (Sezneva 2013) ${ }^{8}$. Un nouveau cahier des charges urbanistique est mis en place par les pouvoirs locaux pour gérer " l'héritage » allemand de la ville (Ibid.). La chute de l'URSS entraîne une désorganisation politique importante. Une opportunité saisie par les pouvoirs locaux à Kaliningrad qui en profitent pour exprimer l'identité allemande de leur territoire mais aussi se rapprocher des voisins européens et gagner en autonomie face au Kremlin, pas toujours bien perçu dans l'exclave. Une opportunité également saisie par d'autres acteurs. L’Université fédérale de la Baltique Emmanuel Kant [Baltijskij federal'nyj universitet im. I. Kanta] de Kaliningrad, nommée en l'honneur du philosophe (et anciennement Université russe d'État E. Kant [Rossijskij gosudarstvennyj universitet imeni I. Kanta]) a été l'un des acteurs principaux de la réhabilitation intellectuelle de la mémoire de Königsberg. À travers différents départements de recherches (dont un champ "d'études kantiennes »), elle a progressivement permis à cette histoire oubliée de force de réémerger, en en faisant l'un de ses marqueurs identitaires principaux, ainsi qu'en se réclamant héritière de l'Université Albertina de Königsberg. En témoigne son nom, qui exprime aujourd'hui une identité plus régionale (Université fédérale balte) qu'auparavant (Université russe d'État). Ainsi s'ouvre une décennie de réouverture historiographique et de

7 Commentaire d'Ina, guide touristique de la ville, relatif à l'avenue Lénine (traduit du russe), visite de terrain, 13 mai 2019.

8 Entretien avec Olga Sezneva, sociologue spécialisée en sociologies culturelles et urbaines et spécialiste de Kaliningrad, Université d'Amsterdam, Pays-Bas, 19 juin 2019, via vidéoconférence depuis Bruxelles. 
réaménagement de la ville?

\subsection{Reconstruction d'un héritage culturel}

Königsberg, ville riche et dense, incarnait le développement économique et marchand insufflé par la Ligue Hanséatique. Plus encore que pour sa prospérité, la ville est connue pour ses figures intellectuelles dont la tête de proue est Emmanuel Kant ${ }^{10}$. Déracinées, les populations installées à Kaliningrad sont privées de leurs terres de naissance. Une partie de ces populations s'installe sur base volontaire à Kaliningrad, suite notamment à la destruction de leurs maisons et villages. Plus de $75 \%$ des nouveaux arrivants sont d'origine paysanne. Ils s'imaginent alors (en 1946-1947) s'installer en Allemagne ou en Prusse, plus qu'en URSS. Les vagues de peuplement suivantes sont en partie forcées et trompées par les campagnes d'information soviétiques qui vantent les opportunités de ce territoire et promettent une reconstruction grâce à d'énormes budgets. Les autorités offrent aux nouveaux arrivants des habitations, bien souvent en ruines, et un budget pour s’installer et reconstruire ce qui peut l'être. La réalité qui les attend est pourtant tout autre. La reconstruction fut lente et très partielle, de nombreux chantiers d'état pharaoniques abandonnés en cours de route, offrant des conditions de vie précaires aux nouveaux arrivants (Eaton 2013). La rencontre de trois facteurs permet alors de poser les bases d'une appropriation culturelle qui ne s'exprimera réellement qu'après la chute de l'URSS : un imaginaire " d'installation en Prusse » chez les nouveaux arrivants ; la vie prolongée de ces nouveaux arrivants dans ce qu'il restait alors des infrastructures et habitations de Königsberg, qui se sentent abandonnés par Moscou à cause de l'inefficacité de la reconstruction ; enfin, la rencontre de ces derniers avec certaines familles allemandes demeurant encore sur place (avant leur expulsion) (Ibid.). Autant de facteurs qui fondent la singularité actuelle de la population de l'exclave russe et son rapport à son histoire, quatre à cinq générations plus tard. À ces facteurs démographiques s'ajoutent des considérations économiques et politiques. Frappée de plein fouet par l'ouverture au marché en 1991 et victime de la vétusté de ses infrastructures, l'industrie locale s'effrite. Isolée du reste de la Russie, mais située aux portes d'une Union européenne en plein développement, la région de Kaliningrad entreprend de saisir une double opportunité : se démarquer politiquement et culturellement de la Russie centrale et orientale d'abord, et reconvertir l'économie locale grâce au tourisme en ouvrant ses portes aux investisseurs et touristes européens ensuite.

9 Ce processus se prolongera après les années 2000, malgré la modification des rapports de force entre autorités locales et fédérales, mais selon des dynamiques différentes (voir titre 3.3)

10 La philosophe Hannah Arendt a également passé une partie de son enfance à Königsberg. Toutefois, aucune référence à sa vie n'est visible dans la ville actuelle de Kaliningrad. 
Quelques monuments ont survécu, en tout ou en partie, aux destructions de la guerre. Labellisées " patrimoine de la culture mondiale » par les Soviétiques, quelques statues ont pu être conservées dès 1946. C'est notamment le cas de la statue de Friedrich Schiller, poète, écrivain et icône de la culture allemande. Une statue à son effigie avait été installée devant le grand théâtre de la ville. Après leur arrivée, des soldats soviétiques taguent le monument «culture mondiale » [mirovaja kul'tura] et entérinent sa conservation ${ }^{11}$. La statue fait aujourd'hui face au Théâtre dramatique [Dramatičeskij teatr], reconstruit selon les standards soviétiques. La statue d'Emmanuel Kant, qui n'a que rarement quitté Königsberg de sa vie, a également été conservée et sera restaurée et réinstallée symboliquement en ville en 1991, ainsi que sa tombe, annexée à la cathédrale de Königsberg en 1924 (voir Figure 6). Une statue de «buffles qui s'affrontent » [Skul'ptura "Borjuščiesja zubry »], offerte par Berlin à Königsberg au début du XX siècle, a également été maintenue et est fièrement exhibée sur l'avenue de la Paix par les guides touristiques locaux.

La cathédrale de Königsberg, à laquelle est adossé le tombeau de Kant, a dû être reconstruite. La rénovation progressive de l'édifice s'est déroulée tout au long des années 1990 (Cybriwsky 2013 ; Костяшов 2016), incarnant parfaitement le processus de rematérialisation del'identité de Königsberg dans les espaces urbains et notamment sur l'île de Kneiphof, rebaptisée « île de Kant » [Ostrov Kant]. Initiée par les pouvoirs et acteurs locaux dès les années 1990, mais en partie reprise par les autorités fédérales au tournant des années 2000, la réhabilitation de «l'héritage » de Königsberg répond autant à une singularité démographique et historique qu’à une tentative politique de démarcation vis-à-vis de la Russie centrale et orientale. Un phénomène dont l'impact sur les identités locales apparaît important et qui s'illustre, au-delà de l'architecture de la ville, par certaines pratiques. Une véritable " économie de l'excavation » est née sur le territoire de l'oblast (Sezneva 2007). De nombreuses personnes s'adonnent en effet aujourd'hui à des recherches, en fouillant le sol, et collectionnent les objets divers datant de l'époque allemande qu'ils découvrent, pour les exposer chez eux, les vendre sur de petits marchés improvisés, ou les faire découvrir à certains touristes nostalgiques, dont les descendants des Allemands ayant fui ou ayant été expulsés du territoire (Ibid.).

\subsection{Königsberg comme parc thématique}

L'expérience touristique de la visite de Kaliningrad en 2019 est particulièrement historicisée. L’avenue Lénine, qui s’étend de la place de la Victoire à la Maison

11 Entretien avec Stefan Berger, le 11 juin 2019, via vidéoconférence depuis Bruxelles. 
des Soviets, renforce cette observation. Si l'avenue a tout de soviétique, elle donne pourtant à voir la stratégie de redéveloppement économique régional. Les façades des immeubles de cinq étages des années 1950-1960 ont été refaites, dans un style "néo-hanséatique ». Le plâtre et les couches de peinture colorée ne trompent toutefois pas l'œil de l'observateur critique. Véritable ersatz touristique, ce réaménagement de façade se prolonge dans ce qui deviendra le "centre historique » de la ville. Sur le site de l'office du tourisme comme sur les sites des agences locales de promotion de la ville, c'est le «Village des Pêcheurs » [Rybnaja Derevnja] qui domine. Sa construction débute en 2005, grâce à un promoteur privé rejoint par les autorités locales - qui bénéficiaient d’importants subsides fédéraux de rénovation urbaine dans le cadre des « 750 ans de Kaliningrad » cette année-là, à l'instar de Saint-Pétersbourg deux ans plus tôt - (Tétart 2007).

Le projet de construction du Village des Pêcheurs, érigé sur l'une des berges de la Pregolja où les barges débarquaient leurs marchandises à Königsberg, porte le nom de « centre ethnographique de loisirs et de commerce » [ètnografičeskij centr otdyha i torgovli]. Un nom qui en dit long sur le résultat de ces aménagements : celui d'un parc thématique valorisant une atmosphère "d'un autre monde " (Sezneva 2013, 778). Comme le souligne Olga Sezneva :

[...] comme méthode de redéveloppement, la thématisation exprime un avantage peu reconnu mais néanmoins distinct : il accueille l'invocation de l'étranger sans pour autant menacer le national. (Ibid.).

[...] si la globalisation peut constituer un danger potentiel pour les identités nationales, Kaliningrad constitue un contre-exemple. L' «autre » y est modélisé comme différent mais compatible avec soi (Sezneva 2013, 779).

Une particularité dans le développement de ce projet et de l'identité locale de Kaliningrad qui semble essentielle au début des années 2000, suite à la volonté de Vladimir Poutine de recentralisation et d'affirmation de l'identité russe des territoires aux marges de la Fédération.

Le parc thématique " Königsberg » à Kaliningrad ne s'arrête pas au Village des Pêcheurs. En effet, les hébergements se sont affublés d'une identité similaire. Plusieurs complexes hôteliers modernes, installés au bord de l'eau, imitent des façades hanséatiques avec une certaine largesse architecturale. Sur l'application Airbnb, des logements sont apparus principalement depuis l'annonce de la sélection de Kaliningrad comme une des villes-hôtes de la Coupe du monde de football 2018, et une bonne partie d'entre eux ne sont disponibles que lorsque l'on recherche la ville de "Königsberg » plutôt que Kaliningrad. Des fresques murales et des restaurants d'ambiance prolongent l'expérience immersive dans la 
ville prussienne. À l'office du tourisme, tous les moyens sont bons pour valoriser la mémoire de Königsberg, y compris la vente du livre d'un journaliste local, Igor Rudnikov, intitulé La République de Königsberg [Respublika Kenigsberg]. Considéré comme " prisonnier politique » par Amnesty International (Amnesty International 2018) et présenté à la fin de son livre par une photo derrière les barreaux, l'auteur semble faire partie lui aussi du package thématique voulu par la stratégie touristique de la ville... au risque de froisser le Kremlin. Outre les hébergements et la littérature, ce sont presque exclusivement des expériences touristiques à travers la mémoire allemande qui sont proposées, déclinées en autant de visites guidées. Le consumérisme valorisé par les autorités locales passe par une double porte : une fascination pour Königsberg opérée par un réaménagement germanisé de la ville et une « idéologie du circuit touristique » censée emmener la ville dans l'ère de la globalisation ${ }^{12}$. Le succès de cette stratégie touristique, comparé à la ville voisine de Gdansk en Pologne, est toutefois relatif. Ayant été complètement reconstruite à l'image de son passé dès les années 1950, cette dernière offre une expérience touristique connaissant, elle, un énorme succès (Kolossov et Radvanyi 2014). (voir Figure 7).

Le Château de Königsberg, un destin incertain (voir Annexe :Vignette 1)

\section{Entre " Kantgrad » et " Severnaja Rus' » : matérialiser une appartenance commune post-soviétique}

Avec l'effondrement de l'Union soviétique, toute la géographie politique de l'Europe centrale et orientale est redessinée. Un processus qui s'accompagne d'une nécessité sociale et politique qui s'exprimera dans l'espace : construire un sentiment d'appartenance commun autour d'une nouvelle organisation politique et territoriale. L'exclave, plus isolée encore par la fin de l'Union soviétique, ne sera rattrapée par ce processus d'affirmation nationale qu'une décennie plus tard, laissant les débats identitaires locaux faire rage, à l'instar de nouveaux noms proposés pour la ville, témoignant d'un gradient d'appropriation de l’identité nationale qui diffère (Tétart 2007, 301-302). La ville, du fait de sa distance par rapport à Moscou et sa proximité directe avec d'autres États devenus membres de l'Union européenne - la Lituanie et la Pologne - en 2004, constitue un territoire aux enjeux singuliers pour le gouvernement russe. Le processus de construction

12 Il est intéressant de noter que la Kaliningrad soviétique était également présentée par les autorités de l'époque à travers de nombreux tours guidés, comme me l'a confié Jamie Freeman. Elles utilisaient cette méthode pour valoriser l'aménagement de la ville auprès des habitants et ajouter à l'influence des infrastructures sur les modes de vie un discours qui se veut performatif. À travers deux époques différentes, on procède ainsi de la même manière pour faire « table rase » : des visites guidées de la « ville nouvelle » sont mises en place. 
des identités nationales de la Russie contemporaine y est inscrit dans les espaces urbains à l'initiative des autorités fédérales et des acteurs locaux qui leurs sont loyaux, essentiellement depuis le début des années 2000 et le retour d'un contrôle accru du Kremlin sur l'enclave. Après la matérialisation des identités soviétique et allemande à Kaliningrad, une troisième « couche » de ce palimpseste urbain se forme, une troisième ville dans la ville : l'identité nationale russe et ses aspects matériels.

\subsection{La réorganisation de l'héritage soviétique}

Le premier élément identitaire et matériel de l'appartenance nationale russe à Kaliningrad est sans aucun doute la réappropriation et la légitimation de son histoire soviétique. Une tendance dont l'acteur principal est le Kremlin, mais dans laquelle interviennent également des associations patriotiques locales. En 2000, la première élection à la présidence russe de Vladimir Poutine marque l'instrumentalisation de narrations de l'histoire soviétique au service du sentiment d'appartenance nationale et de la légitimité du nouveau pouvoir fédéral. Un monument à la gloire d'Aleksandr Marinesko, officier de la marine soviétique, est inauguré en 2001 dans la ville sous l'appui d'une association locale d'anciens combattants. L'imposante statue de Lénine sur la place de la Victoire sera en revanche déplacée, pour faire place à la « colonne de la Victoire » [Kolonna Pobedy] (installée en 2005), célébrant la victoire soviétique lors de la Seconde Guerre mondiale. Fort du souvenir de la victoire soviétique sur les Allemands, le maréchal Vasilevskij trouve également sa place au nord de ville (installée en 2000), lui qui dirigeait le régiment ayant libéré la ville en 1945. Autant de monuments qui permettent d'associer la victoire lors de cette guerre à l'identité russe, en réorganisant le souvenir soviétique. La Première Guerre mondiale rejoint de manière plus surprenante les représentations militaires soviétiques dans la matérialité de la ville en $2014^{13}$. Sorte de surenchère au parc de la Victoire aménagé par les Soviétiques en 1945, un monument colossal, l'un des plus imposants de toute la ville, a été installé juste à côté, à l'extérieur du parc. Celui-ci rend hommage au nom de la Russie aux combattants de la Première Guerre mondiale. Dans le même ordre d'idées, un nouveau monument à la Seconde Guerre mondiale est installé au sein même du parc de la Victoire et rejoint l'ensemble mémoriel déjà aménagé à l'époque soviétique.

La réorganisation de l'héritage matériel soviétique et des narrations qui l'accompagnent s'apparente dans son expression contemporaine à un assemblage.

13 Plus surprenante, dans la mesure où la Première Guerre mondiale est généralement associée à une défaite et une humiliation en Russie, et donc effacée du récit national contemporain. Entretien avec Stefan Berger, le 11 juin 2019, via vidéoconférence depuis Bruxelles. 
L'histoire soviétique est mobilisée comme un élément clé du récit national de la Russie de Vladimir Poutine, censé générer un sentiment de légitimité et d'appartenance. Ces différentes références historiques sont organisées selon un dessein particulier, décrit par l'anthropologue Michel Bouchard comme la « curation de la nation » (Bouchard 2013). Véritable sélection arbitraire d'éléments historiques, le projet national russe de Vladimir Poutine mobilise des narrations qui lui sont propres. Un processus qui, en plus de permettre l'instrumentalisation d'éléments historiques au profit de la stimulation du sentiment d'appartenance nationale, permet également d'en "panser les plaies » en aménageant certains traumas collectifs de la population (Ibid.). Ce processus de curation de la nation remobilise le sentiment de fierté soviétique, ainsi que les « victoires » militaires associées, mais en désidéologise le style pour le rendre compatible avec les « nouveaux» attributs de la nation russe (Rethman 2008). L'exemple parfait est probablement la « Mère patrie » [Rodina mat'], personnification soviétique représentée par de nombreuses statues en URSS, inaugurée en 1974 à Kaliningrad. Elle constitue une autre figure notable de la réorganisation des symboles de l'époque. Elle est en effet aujourd'hui rebaptisée " Mère Russie » [Mat' Rossija] à Kaliningrad et nourrit la nouvelle symbolique nationale.

\subsection{Les « nouveaux » héros}

En parallèle au reste du territoire russe, la réaffirmation de Kaliningrad comme partie intégrante du système d'appartenance nationale a mené à la présence de « nouveaux » héros nationaux. Non que ceux-ci aient tous vécu récemment, mais leur présence dans la ville de Kaliningrad est inédite. " La légitimité d'une nation relève de la préservation d'un héritage » (Thiesse 1999). Ainsi, pour construire l'appartenance à la communauté nationale, le poids du patrimoine symbolique de supposés ancêtres permet de relever une forme de respect et de préservation d'une union qui dure depuis longtemps. C'est cette durée en particulier qui insuffle la croyance d'un besoin de conserver pour continuer à donner du sens à l'entité nationale à laquelle on appartient (Ibid.). Ainsi, le monument représente particulièrement bien cette inscription dans la durée d'une mémoire atemporelle, et semble être l'un des outils privilégiés pour signifier une intention de conserver des traditions nationales. Réifiée en divers héros dont l'importance est construite par le projet politique et discursif de consolidation de la nation, la construction identitaire nationale se matérialise dans des monuments qui constituent à eux seuls les représentations d'une promesse faite à soi-même : pérenniser la mémoire de ces héros qui stabilisent l'identité de la communauté nationale. 
Ainsi, dès les années 2000, les héros russes font leur apparition dans la ville de Kaliningrad et incarnent différents attributs de l'identité nationale. Alexandre Nevski est l'un d'eux. Prince russe du XIII ${ }^{\mathrm{e}}$ siècle, guerrier sanctifié par l'église orthodoxe, il est l'un des héros nationaux les plus plébiscités (Bouchard 2013) ${ }^{14}$. Au nord-est de Kaliningrad, une impressionnante statue à son effigie, posée sur une épaisse colonne de pierre, fut inaugurée en 2018 et immédiatement bénie par le clergé local. Sur l'île de Kant autant que sur l'avenue de la Paix, c'est le Tsar Pierre $1^{\text {er }}$, dit Pierre le Grand, qui est représenté à travers des monuments (inaugurés respectivement en 2017 et 2003), symboles de ses " nombreuses visites à Königsberg » et des prétendues « racines slaves » du territoire jadis chères à Staline.

Cette nouvelle affirmation nationale s'exprime dans l'un des noms que la ville aurait pu recevoir à la chute de l'Union soviétique. " Severnaja Rus' », soit la " Rus' du Nord », c'est le nom proposé par le président de la Douma fédérale russe en 1991, voulant rappeler à Kaliningrad son appartenance nationale (Tétart 2007, 302). Une proposition qui ne fera toutefois pas le poids face à l'inertie de l'héritage soviétique, représenté par le nom actuel de la ville, Kaliningrad (voir Figure 10).

Le pilier national orthodoxe (voir Annexe : Vignette 2)

\subsection{L'étranger au service du national}

La matérialisation du récit national russe est un projet d'État. Néanmoins, une stratégie spécifique répond à la singularité de l'enclave de Kaliningrad. Témoin de l'appropriation croissante du souvenir de Königsberg par les acteurs locaux, le gouvernement fédéral russe entreprend lui aussi d'en faire usage au tournant des années 2000. Le Kremlin entend alors présenter cette mémoire comme compatible avec les identités nationales. Thématisé, mis en tourisme et simulé, le souvenir de Königsberg compose une expérience à haut potentiel économique et identitaire. L'histoire de Königsberg est exploitéeà Kaliningrad pour « rafraîchir » le branding identitaire national. Une stratégie parfaitement représentée par une fresque faisant partie du projet originel du Village des Pêcheurs, qui indiquait sur l'une des façades : « la racine prussienne de l'arbre russe » [ prusskij koren' russkogo dereva »] (Sezneva 2013, 781). L'histoire prussienne, soumise à l'histoire russe, devient en quelque sorte un attribut national « exotique » du fait de sa spécificité culturelle, mais compatible avec le récit national russe, qu'elle vient même légitimer et nourrir (Ibid.). En témoigne également l'édition récente d'une

14 Alexandre Nevski est un héros national parfois mobilisé à l'ère soviétique (Bouchard 2013). Néanmoins, il prend une place plus importante dans la ville post-soviétique à Kaliningrad. 
carte touristique de la ville pour le Mondial de football, sur laquelle un encart indique :

[...] Emmanuel Kant est né et a vécu à Königsberg. Il est intéressant que lui aussi puisse être considéré comme un sujet de l'Empire russe : en 1758, pendant la guerre de Sept Ans, les troupes russes sont entrées dans Königsberg, et les résidents locaux ont juré fidélité à l'impératrice Élisabeth. Le philosophe était parmi eux.

L'anniversaire des « 750 ans » de Kaliningrad (voir Annexe : Vignette 3)

Dès la chute de l'URSS en 1991, de nombreuses villes sont renommées. Leurs noms soviétiques disparaissent au profit de leurs noms précédents ou de nouveaux noms plus russes. Que faire dans le cas de Kaliningrad ? Si un retour au nom de Königsberg est proscrit, essentiellement à cause des avis défavorables d'associations d'anciens combattants, des propositions émergent, telles « Kantgrad ${ }^{15}$ ou « Mont Royal » ${ }^{16}$ [Korolevskaja Gora]. Néanmoins, aucune des propositions ne convainc les autorités fédérales. Le risque de compromettre l'identité russe de la région est trop important (Tétart 2007, 301-302). Qu'en estil aujourd'hui, au vu de l'appropriation croissante, locale mais aussi nationale, de l'identité prussienne du territoire ? La mobilisation de l'histoire soviétique et de l'image de trophée de guerre de Kaliningrad semble prendre le pas sur l'opportunité touristique «Königsberg » aux yeux des autorités fédérales, mais combien de temps résistera-t-elle à l'intérêt croissant des pouvoirs locaux pour l'histoire allemande?

\section{4. "Kenig » : vers l'émergence d'une identité locale dissonante?}

"Il faut seulement comprendre qu'une friche, ce n'est pas l'absence de paysage, mais bien un espace figé entre ce qüil n'est plus et ce quïl n'est pas encore ${ }^{17}$.

Trois identités, territorialisées et temporalisées, se rencontrent à Kaliningrad par leur matérialisation dans les aménagements urbains. L'identité soviétique rencontre l'identité allemande, en partie réhabilitée, et l’identité nationale russe. À travers ces différentes identités et les espaces où elles s'expriment, se construisent des appartenances collectives et une mémoire sociale propre aux communautés qui habitent la ville. Quelles identités collectives sont formées, reformées ou déformées par les représentations matérielles qui habitent leurs déplacements quotidiens? Au vu des incompatibilités historiques de certaines identités territoriales à Kaliningrad, les identités collectives locales ont-elles

15 Référence à Kant, mais sous la forme « classique » du nom d'une ville russe. Littéralement : « KantVille ».

16 Traduction littérale de Königsberg en russe.

17 Aleksandra Artamonova, artiste originaire de Kaliningrad (Артамонова 2018). 
un aspect dissonant ? Le projet nationaliste russe, particulièrement attentif à Kaliningrad du fait de sa position géographique, est-il en train de faire à nouveau table rase pour maintenir un sentiment d'appartenance nationale supérieur à toute autre forme d'identification ? La mondialisation est-elle en train de lisser la mémoire régionale au profit d'identités globalisée? Les autorités locales parviennent-elles à se ménager une autonomie et une singularité croissante ? Ces questions méritent que l'on s'intéresse de manière transversale à la formation des identités collectives à Kaliningrad.

\section{1 « Kenig » : compromis ou aliénation ?}

Après Königsberg et Kaliningrad, la ville porte un nouveau nom. Bien qu'il ne soit pas officiel, il est très utilisé et représentatif des questionnements identitaires que traverse ce territoire. Il s'agit de « Kenig », forme raccourcie de Königsberg. Cette nouvelle appellation populaire semble être le signe d'une réappropriation de l'histoire pré-soviétique de la ville par ses habitants actuels, qui ne l'ont pourtant jamais vécue, tout en continuant d'exprimer des attributs identitaires et culturels russes ${ }^{18}$ (Berger 2010 ; Sezneva 2013). Plusieurs éléments caractérisent les identités collectives contemporaines à Kaliningrad.

Premièrement, le retour de l'identité allemande tient à un sentiment de déracinement, effectif sur plusieurs générations (Sezneva 2013). Le repeuplement de Kaliningrad en 1946 et le refus du souvenir du passé allemand simultané ont eu pour conséquence un besoin de construire sur le long terme les identités collectives en recouvrant une forme d'appartenance et une identité aliénée. Les populations transmigrées ainsi que celles installées sur base volontaire à Kaliningrad viennent de nombreuses régions russes (ou biélorusses). Leurs villages ont bien souvent été rasés pendant la Seconde Guerre mondiale (Eaton 2013). Ayant pratiquement tout perdu, Kaliningrad constitue pour ces populations une possibilité de se reconstruire une nouvelle vie, ainsi qu'une terre prussienne conquise et " méritée par les souffrances de la guerre », qui est maintenant à leur disposition (Eaton 2013). Pour pallier la perte de leurs racines, mais aussi grâce à la rencontre des populations russes réinstallées avec les quelques populations allemandes restantes en 1946-1947 (Eaton 2013), les habitants de Kaliningrad se réapproprient personnellement et collectivement la mémoire de Königsberg, rendue accessible par la chute de l'Union soviétique (voir Figure 13).

Deuxièmement, aujourd'hui le passé allemand offre une opportunité identitaire ayant un impact économique probant. Ainsi, tant les pouvoirs publics

18 À l'instar de Saint-Pétersbourg, surnommée « Piter » par ses habitants, qui redécouvre son histoire tsariste présoviétique. 
que les citoyens de la ville mobilisent cet atout identitaire pour se distinguer sur le marché du tourisme (destiné au reste de la Russie et aux pays étrangers) et sur le marché des services destiné aux locaux (en témoignent les nombreux commerces qui adoptent cette étiquette identitaire : « Königs Optik », « KenigAvto », « Kenig Hair », « Kenig Doors », « Königsbacker », etc.) (Tétart 2007 ; Siegien s.d.). Cette opportunité économique agit comme facilitateur à l'appropriation collective d'une identité territoriale prussienne.

Troisièmement, l'identification à l'histoire allemande correspond également à un besoin d'exprimer une particularité locale rendant plus justifiable ou compréhensible la situation géographique et politique complexe de l'enclave. Forme de retournement de stigmate, l'identité allemande vient compenser les conséquences difficiles de l'enclavement pour ses habitants en mobilisant une particularité historique.

Enfin, l'identité allemande constitue une porte d'accès à un autre attribut identitaire : le caractère " européen » de Kaliningrad et ses habitants. Non que la ville simule une identité européenne, mais l'identité allemande permet de légitimer cet attribut dont les définitions sont nombreuses et font débat, autant en Europe de l'Est que de l'Ouest. L'accès à la mémoire de Königsberg nourrit le sentiment d'appartenance européenne de la ville, qui lui-même est associé à une forme de modernité économique et à un modèle de prospérité et donc à la globalisation. En témoigne l'utilisation fréquente de termes anglais dans les enseignes commerçantes « Kenig ", ainsi que l'utilisation de l'alphabet latin plutôt que cyrillique.

Kant, philosophe « russe » ou trublion local du récit national ? (voir Annexe : Vignette 4)

\subsection{De l'appropriation à la mobilisation}

Au cours des années 1990, la vie politique de Kaliningrad se restructure. Un parti local émerge comme une illustration des relations entretenues entreles différentes identités en présence et leurs relatives appropriations par la population. Parti marginal et peu soutenu, le « Parti républicain balte » [Baltijskaja respublikanskaja partija], devenu «Respublika » au début des années 2000 n'en est pas moins symptomatique des frénésies identitaires engendrées par la compétition des identités russes et prussiennes/allemandes à Kaliningrad.

À l'origine, le parti était dirigé par Sergej Pas'ko, qui ne sera jamais crédité de plus de 5 à $10 \%$ des intentions de vote (Tétart 2007). Celui-ci décède quelques années plus tard, dans des circonstances que son successeur fustige, accusant sur son blog le FSB de l'avoir empoisonné, et documentant les différentes perquisitions 
menées chez lui photos à l’appui (Baltic Republican Party 2019) ${ }^{19}$. Son successeur, c'est Rustam Vasil'ev, curieux personnage aux revendications symboliques d'une appropriation radicale de la mémoire de Königsberg comme contre-identité face au nationalisme russe. Les revendications de Respublika sont essentiellement liées à l'organisation territoriale de Kaliningrad. Premièrement, le parti souhaite l'accès au statut de république, afin de devenir la « quatrième république balte ", tout en restant rattaché à la Fédération de Russie. Une revendication autonomiste mais pas sécessionniste donc. Le deuxième objectif est de revenir au nom de Königsberg pour faire fi des traumas soviétiques rappelés par le nom actuel de l'enclave. Enfin, le troisième objectif principal du parti est de faire une demande d'adhésion à l'Union européenne selon un nouveau statut particulier, créé pour l'occasion, pour désenclaver la région et revenir à un développement économique pérenne (Tétart 2007) ${ }^{20}$.

Ces revendications semblent particulièrement désorganisées et utopiques, voire égocentriques sur le plan des relations internationales. Elles n'en sont pas moins symptomatiques de la possible crise identitaire que certains peuvent traverser face à la multiplicité des attributs identitaires de Kaliningrad. Elles soulignent aussi les dangers que l'identité allemande peut représenter pour le système d'appartenance nationale russe. À force de militantisme et de mobilisation dans les espaces publics, agitant des drapeaux prussiens et des t-shirts floqués « Königsberg », ou encore vendant des Tupperware de «terre prussienne » sur internet pour la « modique » somme de 50 euros, Rustam Vasil'ev a attiré l'attention des autorités et des forces de sécurité. Après plusieurs altercations physiques et de nouvelles perquisitions dans son appartement, suivies d'un départ d'incendie d'après lui criminel, il s'exile à New York et demande l'asile politique. Celui-ci lui aurait été refusé en mars 2018 d’après son blog personnel (Baltic Republican Party 2019). Son parti, lui, est exclu de la vie politique locale depuis l'été 2003 (Tétart 2007, 311).

De l'appropriation culturelle allemande à la mobilisation politique, le cheminement identitaire semble ici passer par une phase d'usurpation, dans la mesure où les membres du parti Respublika n'ont jamais vraiment connu la réalité de Königsberg. Possible appui face à un régime national liberticide et désapprouvé, le cas de Rustam Vasil'ev illustre à la fois le refuge que constitue l'histoire allemande pour les locaux, et l'attractivité de l'identité européenne, vue comme une garantie de prospérité. Ce cas témoigne aussi des moyens sécuritaires

19 Voir aussi un article du blog de Mediapart à ce sujet : Pierre Haffner, "Le FSB contre les régionalistes à Kaliningrad ", Blogs.mediapart.fr, 14 mai 2018.

20 Voir aussi Деловая жизнь

CONNEXE 6 | 2020 - Espace baltique : dynamiques identitaires et stratégies politiques en question(s) 
particuliers mobilisés par le Kremlin pour s'assurer la loyauté de son enclave, reconnaissant par-là que la définition des identités collectives y constitue un enjeu brûlant qui peut facilement échapper à son contrôle.

\section{Conclusion}

En un peu plus de 70 ans, Kaliningrad est passée de trophée de guerre à carte de visite russe en Europe (Tétart 2007, 393). Au-delà de l'apparente complexité géographique de ce territoire, morceau de Russie enclavé au sein de l'Union européenne, de nombreux enjeux locaux cristallisent la reconfiguration identitaire de ce territoire. Il existe une multiplicité de villes dans la ville, à l'image des nombreux noms qu'elle aurait pu porter. C'est toutefois un surnom informel qui souligne particulièrement le rapport à l'espace inscrit dans les identités collectives à Kaliningrad.

« Kenig », forme raccourcie de Königsberg, symbolise la réorganisation par le bas de l'histoire de la ville et la réappropriation par la population de ses ruptures historiques. Si le sentiment d'appartenance russe semble peu discuté (mais indiscutable), il apparaît néanmoins que celui-ci s'hybride à une appropriation croissante des identités allemande et européenne de la ville, néanmoins reconfigurées localement dans leurs significations (Ibid.) (voir Figure 14).

Cet acronyme symbolise pourtant aussi l'assimilation populaire d'un processus de réappropriation culturelle inscrit dans l'espace par les pouvoirs publics. Si le cheminement identitaire opéré par Kaliningrad s'exprime dans la matérialité de la ville, c'est finalement l'incertitude et l'« uchronie ${ }^{21}$ (Carrère 1986) qui y sont les plus visibles. Comme l'écrivait la journaliste et artiste locale Aleksandra Artamonova, « il faut seulement comprendre qu'une friche, ce n'est pas l'absence de paysage, mais bien un espace figé entre ce qu'il n'est plus et ce qu'il n'est pas encore » (Артамонова 2018). Kaliningrad, véritable friche identitaire, demeure sous couverture de son nom soviétique. Mais pour combien de temps?

\section{>> Illustrations}

Je souhaite remercier chaleureusement les professeurs Jean-Michel Decroly, Pieter Lagrou et Mathieu Van Criekingen, de l'Université libre de Bruxelles, pour leur soutien, notamment méthodologique, à mon projet de recherche. Je remercie tout particulièrement la professeure Giulia Prelz Oltramonti (ESPOL, Lille) pour sa confiance et ses conseils.

21 L'uchronie est au temps ce que l'utopie est à l'espace. Ainsi, à travers ses aménagements, Kaliningrad semble ne plus se situer dans une époque, mais dans plusieurs et aucune à la fois. 


\section{Références bibliographiques}

Amundsen, Michael. 2019. "Humanizing the robot: Kaliningrad's House of Soviets and the state of decay." Ambiances [Online], Varia.

Archer, Clive, and Tobias Etzold. 2010. "The European Union and Kaliningrad: Taking the Low Road.” Geopolitics 15 (2): 329-344.

Berger, Stefan. 2010. "How to be Russian with a Difference? Kaliningrad and its German Past." Geopolitics 15 (2): 345-366.

Berger, Stefan, and Paul Holtom. 2008. "Locating Kaliningrad and Königsberg in Russian and German Collective Identity Discourses and Political Symbolism in the 750th Anniversary Celebrations of 2005." Journal of Baltic Studies 39(1): 15-37.

Bouchard, Michel. 2013. « La Grande Guerre patriotique : narrations sociales et monuments de guerre ».Anthropologica 55 (1) : 113-126.

Carrère, Emmanuel. 1986. Le détroit de Behring. Introduction à l'uchronie. Paris : Ed. P.O.L.

Cybriwsky, Roman. 2013. “Königsberg and Kaliningrad: An Historic European City and a Strategic Exclave of Russia." Focus on Geography 56(4): 117-123.

Dementyev, Ilya. 2015. "From 'Ancient Slavialand' to 'Paradise Lost': The Rehabilitation of the Historical Heritage in Kaliningrad (Late 1940s to Early 1980s)," 126-145. In Contact Zones in the Historical Area of East Prussia XXX. Klaipedda: Klaipèdos Universiteto.

Diener, Alexander, and Joshua Hagen. 2011. "Geopolitics of the Kaliningrad Exclave and Enclave: Russian and EU Perspectives." Eurasian Geography and Economics 52 (4): 567-592.

Eaton, Nicole M. 2013. "Exclave: Politics, Ideology, and Everyday Life in KönigsbergKaliningrad, 1928-1948.” Doctoral dissertation, UC Berkeley.

Hirt, Sonia. 2013. "Whatever happened to the (post)socialist city?" Cities, Current Research on Cities 32: 29-38.

Kauffmann, Jean-Paul. 2016. Outre-terre. Paris : Gallimard, Collection Folio.

Kolossov, Vladimir, et Jean Radvanyi. 2014. " Kaliningrad : "l'exclave" russe rêve d'intégration européenne ". Regards de l'Observatoire franco-russe.

Костяшов, Юрий. 2016. “Кёнигсбергский Кафедральный Собор и Могила Иммануила Канта в Советском Калининграде.” Кантовский Сборник 35(4): 79-102.

Kowalczyk, Halina (dir.), et al. 2019. “Fortress Kaliningrad.” OSW Centre for Eastern Studies.

Lebow, Katherine. 2013. Unfinished Utopia: Nowa Huta, Stalinism, and Polish Society, 194956. Cornell University Press.

Le Normand, Brigitte. 2014. Designing Tito's Capital: Urban Planning, Modernism, and Socialism in Belgrade. Pittsburgh: University of Pittsburgh Press.

Rethmann, Petra. 2008. « Nostalgie à Moscou ». Anthropologie et Sociétés 32 (1-2) : 85-102.

Serrier, Thomas. 2010. " Nier ou intégrer l'héritage allemand ? À propos de l'appropriation culturelle de Danzig, Königsberg et Reval à Gdańsk, Kaliningrad et Tallinn ». Revue germanique internationale $11: 223-234$.

Sezneva, Olga. 2007. "We Have Never Been German...”. In Practising Culture, edited by Craig Calhoun and Richard Sennett, 13-34. Routledge. 
Colin HORENBEEK - Paysage urbain et identités collectives à Kaliningrad

Sezneva, Olga. 2013. "Architecture of Descent: Historical Reconstructions and the Politics of Belonging in Kaliningrad, the Former Königsberg.” Journal of Urban History 39 (4): 767787.

Siegień, Paulina. 2016. “How Much Königsberg is in Kaliningrad.” New Eastern Europe 23(5): 115-122.

Tétart, Frank. 2007. Géopolitique de Kaliningrad : une "île » russe au sein de l'Union européenne élargie. Paris : Presses de l'Université Paris-Sorbonne.

Thiesse, Anne-Marie. 1999. La création des identités nationales : Europe XVIIIe-XXe siècle. L'Univers historique. Paris : Ed. du Seuil.

Torbakov, Igor. 2014. "The Russian Orthodox Church and Contestations Over History in Contemporary Russia.” Demokratizatsiya 22 (1): 145-170.

\section{Articles de presse}

Артамонова, Александра. 2018. “ Немота стены ». NovïKaliningrad.ru.

Haffner, Pierre. 14.05.2018. « Le FSB contre les régionalistes à Kaliningrad ». Blogs.mediapart.fr.

Хлебников, Вадим. 2018. « Конец политики ». Новый Калининград.

NoviiKaliningrad.ru. 03.12.2018a. « Алиханов о возможном переезде мэрии в Дом Советов : "Мы там вместе все не поместимся" ".

NoviiKaliningrad.ru. 27.11.2018b. “"Имя врага” : в Калининграде облили краской памятник Канту (фото)»

NoviiKaliningrad.ru. 04.12.2018c. « В конкурсе имен для аэропорта “Храброво” победила Елизавета ".

Ria Novosti. 29.10.2018. “ Аэропорту Калининграда могут присвоить имя Иммануила Канта ».

\section{Sites web}

Amnesty International. 05.07.2018. « Igor Roudnikov, Kaliningrad ».

Baltic Republican Party. Деловая жизнь.

Голос.11.09.2017. « Выборы в Калининграде : подкуп и катастрофический подсчет ».

\section{Entretiens avec des scientifiques}

Stefan Berger, historien de l'Université de Bochum en Allemagne, spécialiste des nationalismes et de Kaliningrad, 11 juin 2019, via vidéoconférence depuis Bruxelles.

Daria Bocharnikova, historienne de l'architecture soviétique, professeure à la KU Leuven, Belgique, 11 juin 2019, Bruxelles.

Olga Sezneva, sociologue spécialisée des sociologies culturelles et urbaines et spécialiste de Kaliningrad, Université d'Amsterdam, Pays-Bas, 19 juin 2019, via vidéoconférence depuis Bruxelles.

Jamie Freeman, historien de l'Université d'East Anglia, Grande-Bretagne, et auteur d'une thèse de doctorat sur les aménagements soviétiques de Kaliningrad, 1 juillet 2019, via vidéoconférence depuis Bruxelles. 


\section{ANNEXE}




\section{Vignette 1}

\section{Le Château de Königsberg, un destin incertain}

voir Figure 8 : Capture d'écran du site de l'office du tourisme de Kaliningrad (page 151)

S’il est un élément du paysage architectural de Königsberg, puis Kaliningrad, qui a subi de plein fouet les ruptures historiques, c'est lui. Le Château de Königsberg symbolisait toute la richesse culturelle et économique de la ville ainsi que ses 700 ans d'histoire. La guerre et la propagande soviétique auront eu raison de son sort. Très endommagé par les bombardements, il demeure toutefois l'un des rares éléments paysagers à ne pas être rasé dans les années suivant l'annexion de la Prusse-Orientale par Staline. Quelques années plus tard néanmoins, son fantôme semble prendre encore trop de place dans la ville. Leonid Brejnev décide de le dynamiter en 1968, et lance le chantier de la Maison des Soviets (Berger 2010 ; Cybriwsky 2013 ; Sezneva 2013) : un fantôme qui en remplace un autre, puisque cette dernière ne sera jamais achevée. Le retour progressif de la mémoire allemande à Kaliningrad relancera l'intérêt et l'admiration pour la bâtisse dans les années 1980. De nombreux projets émergeront dans les années suivantes pour lui redonner vie et ainsi rematérialiser la richesse de Königsberg, réappropriée par la population et les autorités locales. Aucun de ces projets n’a jusqu'à présent vu le jour ${ }^{1}$. S’il n’est pas reconstruit, la volonté de voir son retour s'est transformée en "mirage touristique ». Le Château de Königsberg est présenté sur le site de l’office du tourisme de Kaliningrad parmi les curiosités à visiter de la ville, via une image reconstituée qui semble réelle... Pourtant, il ne reste aujourd'hui plus rien de cette construction, si ce n'est une ouverture dans le sol laissant apparaître quelques briques rouges. À travers ce Château qui n'est plus mais dont le souvenir persiste, la volonté de retour à Königsberg, fruit d'une stratégie économique autant que de la " condition de déplacé » (Sezneva 2013, 783) de sa population, se heurte à la difficulté de faire revivre un héritage si durement balayé dans les années 1940-1950.

voir Figure 9 : Ruines du château de Königsberg (page 151)

1 Entretien avec Jamie Freeman, via vidéoconférence depuis Bruxelles, le 1 juillet 2019. 


\section{Vignette 2}

\section{Le pilier national orthodoxe}

La religion orthodoxe constitue l'un des piliers de l'identité nationale russe promue par le Kremlin (Torbakov 2014). Son assise spatiale est de ce fait particulièrement imposante, et Kaliningrad n'échappe pas à la règle. On retrouve en tête de cette liste la colossale cathédrale du ChristSauveur [Hram Hrista Spasitelja] sur la place de la Victoire, inaugurée en 2005 en présence de Vladimir Poutine (pour les festivités du « $750^{\mathrm{e}}$ anniversaire » de la ville), et devant laquelle les babouchkas défilent en s'arrêtant quelques instants pour faire leur signe de croix. Répondant à la surenchère de monuments dans le parc de la Victoire, on y retrouve également une chapelle orthodoxe... aménagée sur les restes d'une fortification de Königsberg. Il est essentiel de souligner le pouvoir ordonnateur de l'Église orthodoxe dans la construction et le maintien de l'identité nationale russe. À travers son autorité morale et dogmatique auprès de la population, symbolisée par ses lieux de cultes, elle stimule une forme de cohésion au sein de la population et légitime le pouvoir en place en lui assurant une certaine loyauté (Torbakov 2014) ${ }^{1}$. Un pouvoir ordonnateur qui n'est d'ailleurs pas que symbolique ou pieux. Ainsi, derrière la cathédrale du Christ-Sauveur, un complexe a été construit en parallèle, et comprend différents services liés à la cathédrale, comme une crèche ou un gymnase labellisé « orthodoxe ", qui symbolisent l'impact du clergé orthodoxe dans la vie quotidienne des habitants de la ville.

voir Figure 11. Cathédrale du Christ-Sauveur et Colonne de la Victoire (page 154)

1 Il est néanmoins nécessaire, comme le souligne Igor Torbakov, de ne pas penser l'Église orthodoxe russe comme un acteur monolithique et univoque. Si son organisation est particulièrement hiérarchique, cela n'empêche pas l'existence de différentes approches religieuses en son sein, ainsi que des divergences en matière de narrations historiques (Torbakov 2014). 


\section{Vignette 3}

\section{L'anniversaire des « 750 ans » de Kaliningrad}

Cette stratégie de «l'étranger au service du national " (Sezneva 2013) a été couronnée par un événement fêté en grande pompe : « le 750 ${ }^{\mathrm{e}}$ anniversaire de Kaliningrad » en 2005. L'appropriation culturelle et historique est évidente, tant dans le nom que les représentations historiques. Ainsi, en parallèle aux tentatives d'archéologie politique russes visant à prouver une prétendue origine slave du territoire, le tsar Pierre le Grand est représenté par un acteur posé sur la proue d'un navire ouvrant les festivités, derrière un drapeau russe reprenant l'image de l'ancienne Porte du Roi (également représentée sur la Figure 12) (Berger \& Holtom 2008 ; Berger 2010). À l'occasion de cet anniversaire, c'est tout un programme de réaménagement de la ville qui est mis en place, comprenant l'inauguration de différents espaces et monuments (la cathédrale du Christ-Sauveur, le Village des Pêcheurs, le Pont du Jubilé [Jubilejnyj most], le réaménagement de l'avenue Lénine, etc.). Une renaissance, en quelque sorte, pour une ville dont on célèbre l'histoire réécrite, plus vivante, plus riche et plus européenne. Une prise de distance aussi avec les ruptures historiques, pour célébrer une continuité nouvelle. Néanmoins, toutes ces représentations de l'identité allemande doivent s’insérer dans un moule patriotique sous haute surveillance, consacré par la présence du président Vladimir Poutine lors des célébrations et la construction de la cathédrale orthodoxe qui domine la place de la Victoire (Sezneva 2013).

voir Figure 12. Stèle commémorant les « 750 ans » de Kaliningrad en 2005 (page 154) 


\section{Vignette 4}

\section{Kant, philosophe « russe » ou trublion local du récit national ?}

Fin 2018, les autorités fédérales russes lancent un grand vote public à l'échelle nationale. L'objectif : permettre aux citoyens de plébisciter les figures populaires qui représentent le mieux leur région, afin de renommer les aéroports. À Kaliningrad, l’identification à Emmanuel Kant a pris de court les autorités. Alors qu'il ne figurait pas sur la liste de noms proposés au vote ${ }^{1}$, il a dû y être rajouté à force d'être réclamé (Ria Novosti 2018). La possibilité de renommer l'aéroport Hrabrovo de Kaliningrad au nom du philosophe allemand n'a, semble-t-il, pas ravi tout le monde sur place. En témoignent les actes de vandalisme qui ont suivi : la statue de Kant et sa tombe ont été aspergées de peinture, et des mots ont été laissés dénonçant un présumé « caractère antirusse » du personnage. Une peinture qui, paradoxalement, avait été transportée dans une bouteille de cognac " Starij Kenigsberg », soit « Vieux Königsberg », abandonnée sur place... (NoviiKaliningrad.ru 2018b). Cette polémique locale illustre à elle seule l'ambivalence de la stratégie de mobilisation de l'identité allemande, soumise au cadre patriotique et nationaliste, et son impact sur les identités collectives. Le gradient d'appropriation du discours sur l'appartenance nationale fonde des représentations différentes de l'histoire allemande et de ses figures identitaires marquantes. Pour garantir la sécurité de l'identité nationale, l'aéroport sera finalement rebaptisé au nom de l’impératrice Élisabeth (NoviiKaliningrad.ru 2018c).

1 Liste : Ivan Bagramjan, Mihail Barclay de Tolly, Aleksandr Vasilevskij, Kuz’ma Galickij, Ivan Černjahovskij, Aleksandr Pokričkin, Pierre le Grand, l’impératrice Elisabeth Petrovna, Viktor Pacaev, Sergej Snegov. 


\section{Illustrations}

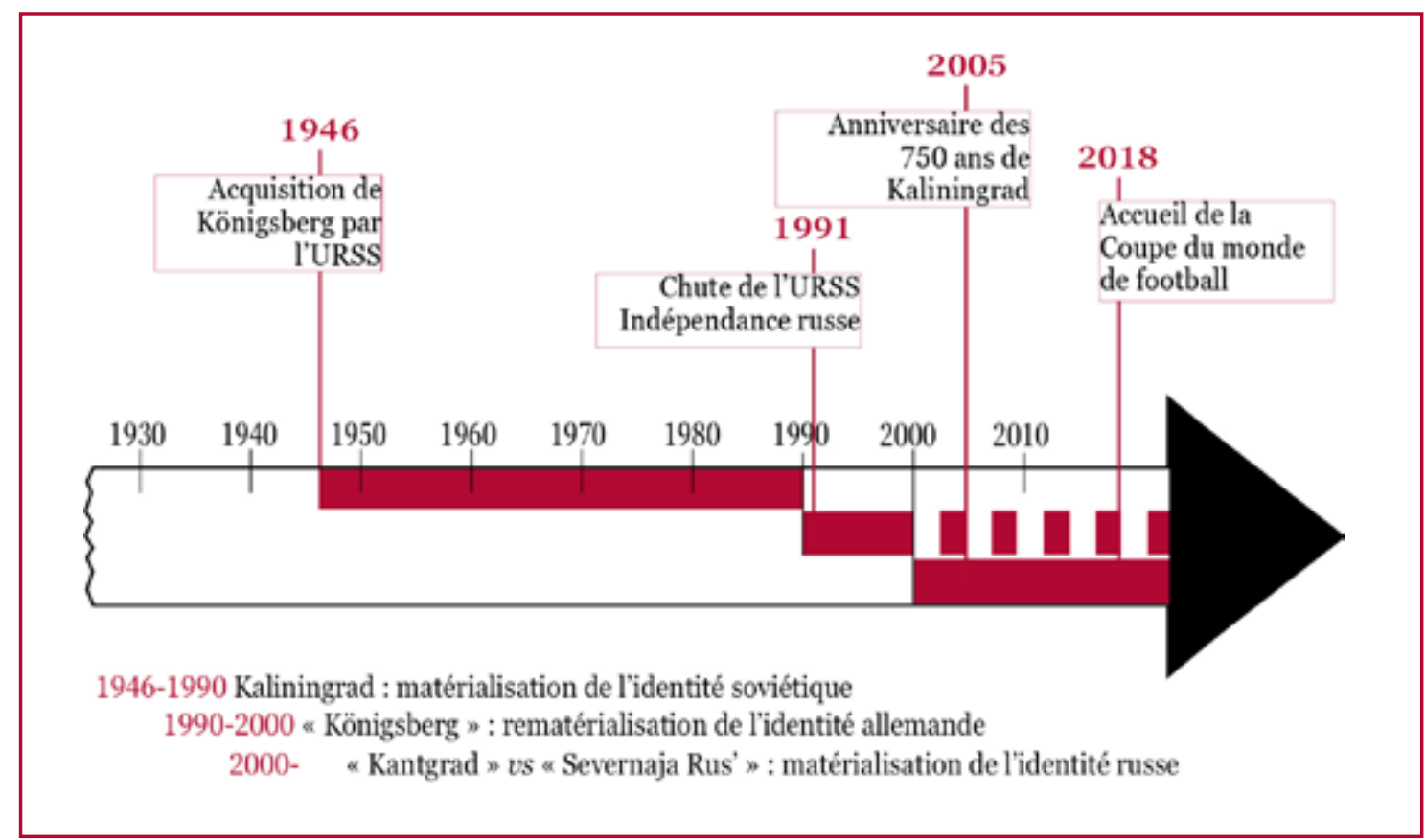

Figure 1. Ligne du temps représentant les reconfigurations identitaires de Kaliningrad depuis 1946, et certains évènements marquants de l'histoire régionale, en parallèle à la structure des chapitres de cet article - (C) Isabelle Renneson, sur base du schéma de l'auteur : Colin Horenbeek, 2020. 

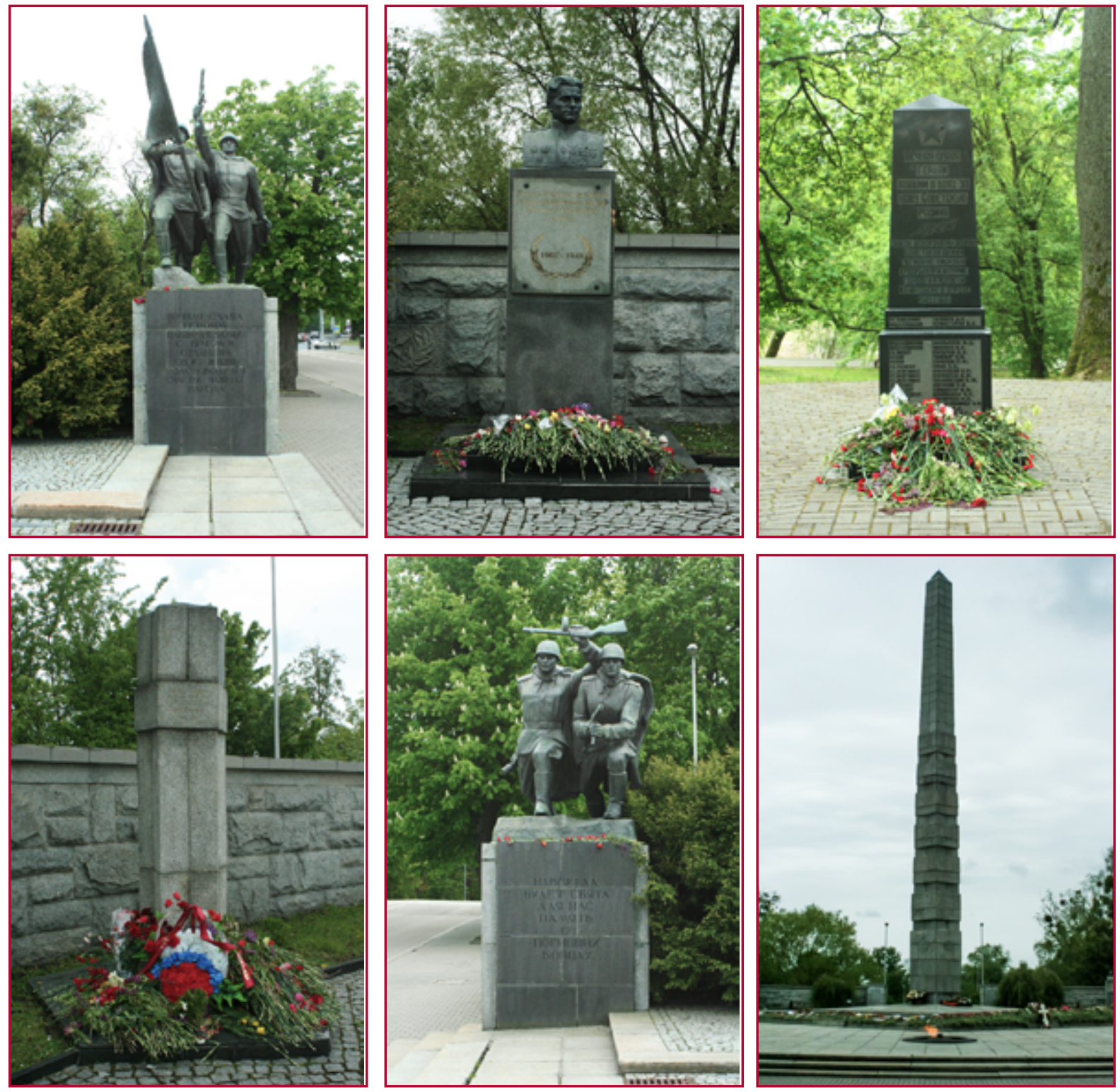

Figure 2. Planche photographique des monuments du parc de la Victoire, construits dès 1945 (non-exhaustif) ; Kaliningrad, mai 2019 - (C) Colin Horenbeek 

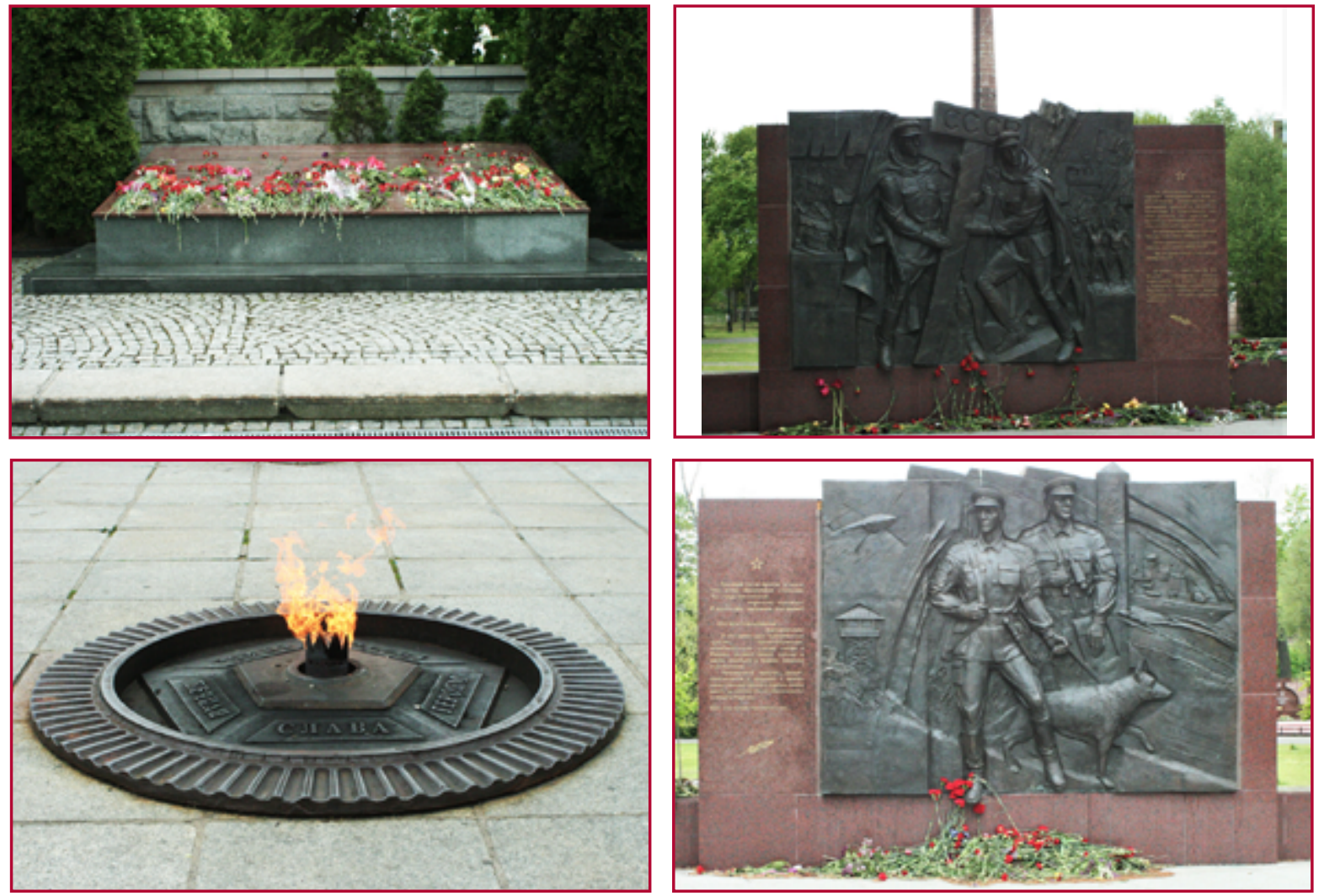

Figure 2. Planche photographique des monuments du parc de la Victoire, construits dès 1945 (non-exhaustif) ; Kaliningrad, mai 2019 - (C) Colin Horenbeek 

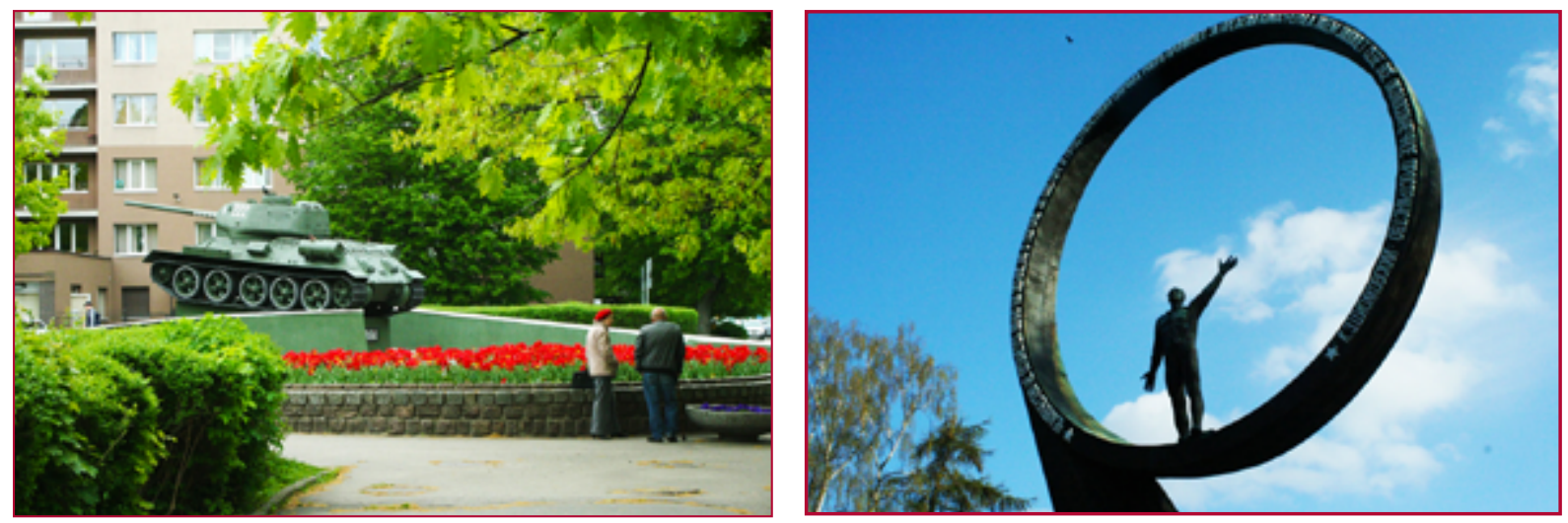

Figure 3. Monument aux Tankistes de la Seconde Figure 4. Monument soviétique aux héros de la Guerre mondiale (1980), installé en plein cour d'un conquête spatiale (1980) ; Kaliningrad, quartier quartier résidentiel, mai 2019 - (C) Colin Horenbeek. d'Amalienau, avril 2018 - (C) Colin Horenbeek.

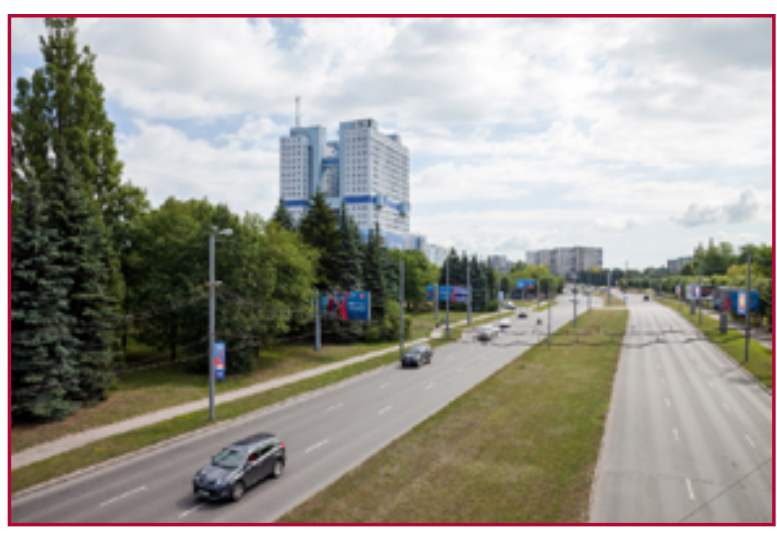

Figure 5. « Dom Sovetov », la Maison des Soviets, qui devait abriter le Soviet suprême de l'URSS, domine les grands boulevards de la ville (Décision de la construction en 1968, début des travaux en 1980); Kaliningrad, juillet 2018 - ๑ Colin Horenbeek.

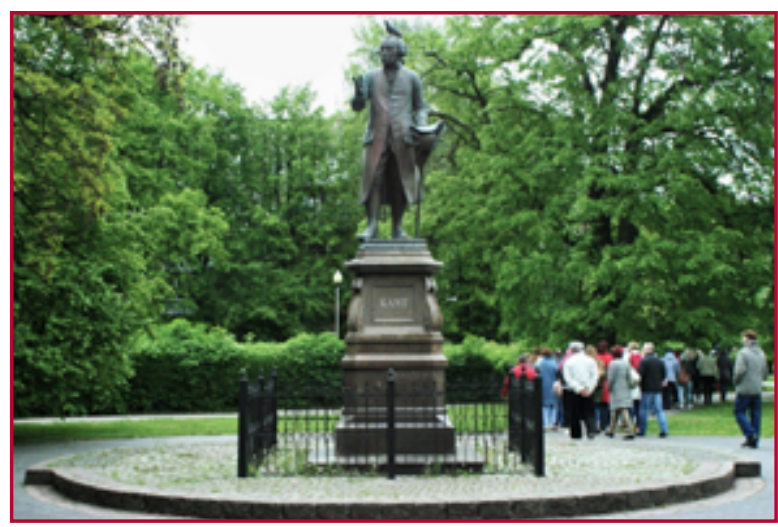

Figure 6. Statue d'Emmanuel Kant (1857, restaurée en 1991), très sollicitée par les visites organisées ; Kaliningrad, mai 2019 - (C) Colin Horenbeek. 

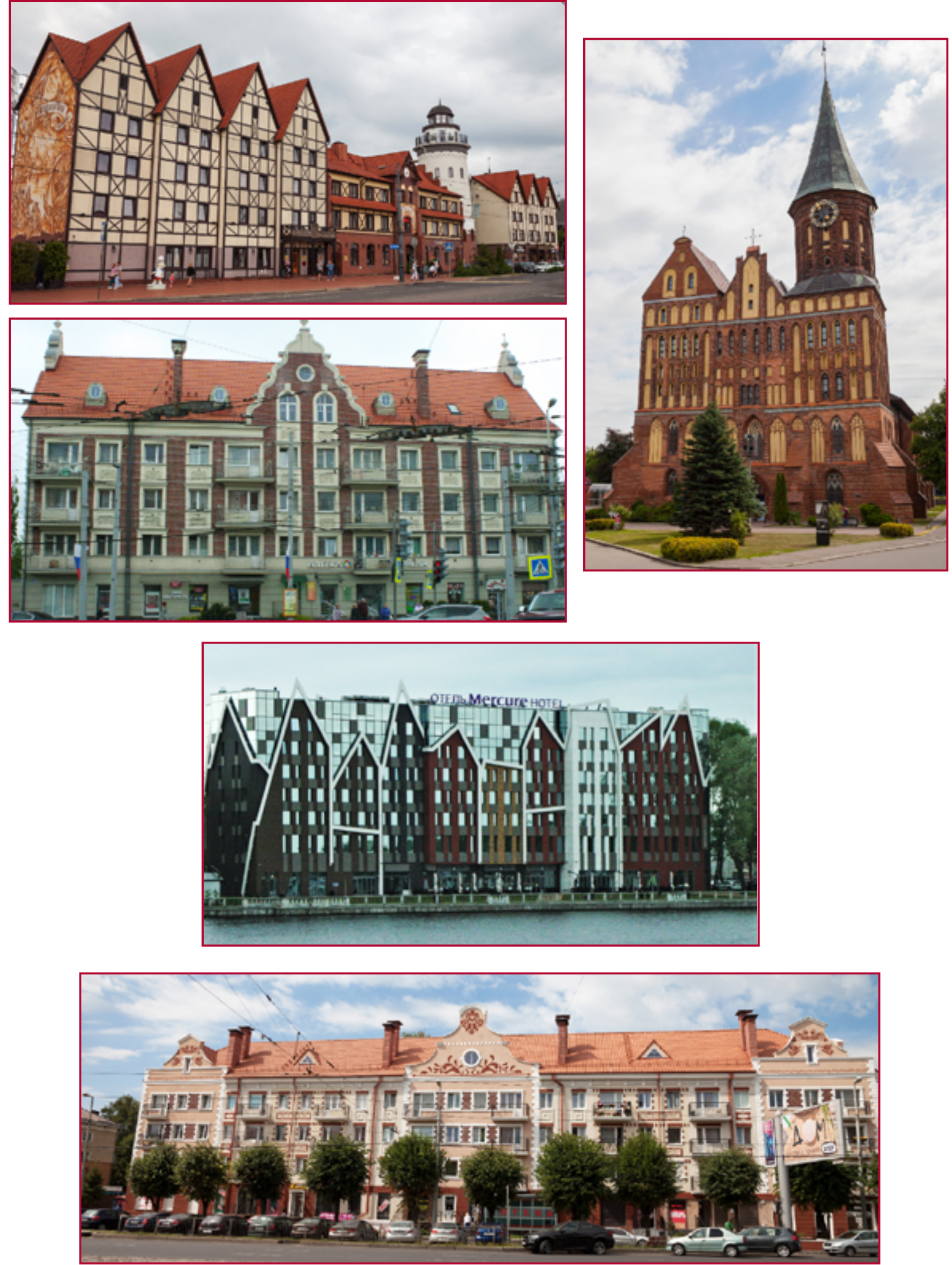

Figure 7. Planche photographique de l'identité allemande de la ville, rematérialisée en parc thématique « Königsberg » dès le début des années 1990 ; Kaliningrad, juillet 2018 et mai 2019 - (C) Colin Horenbeek 

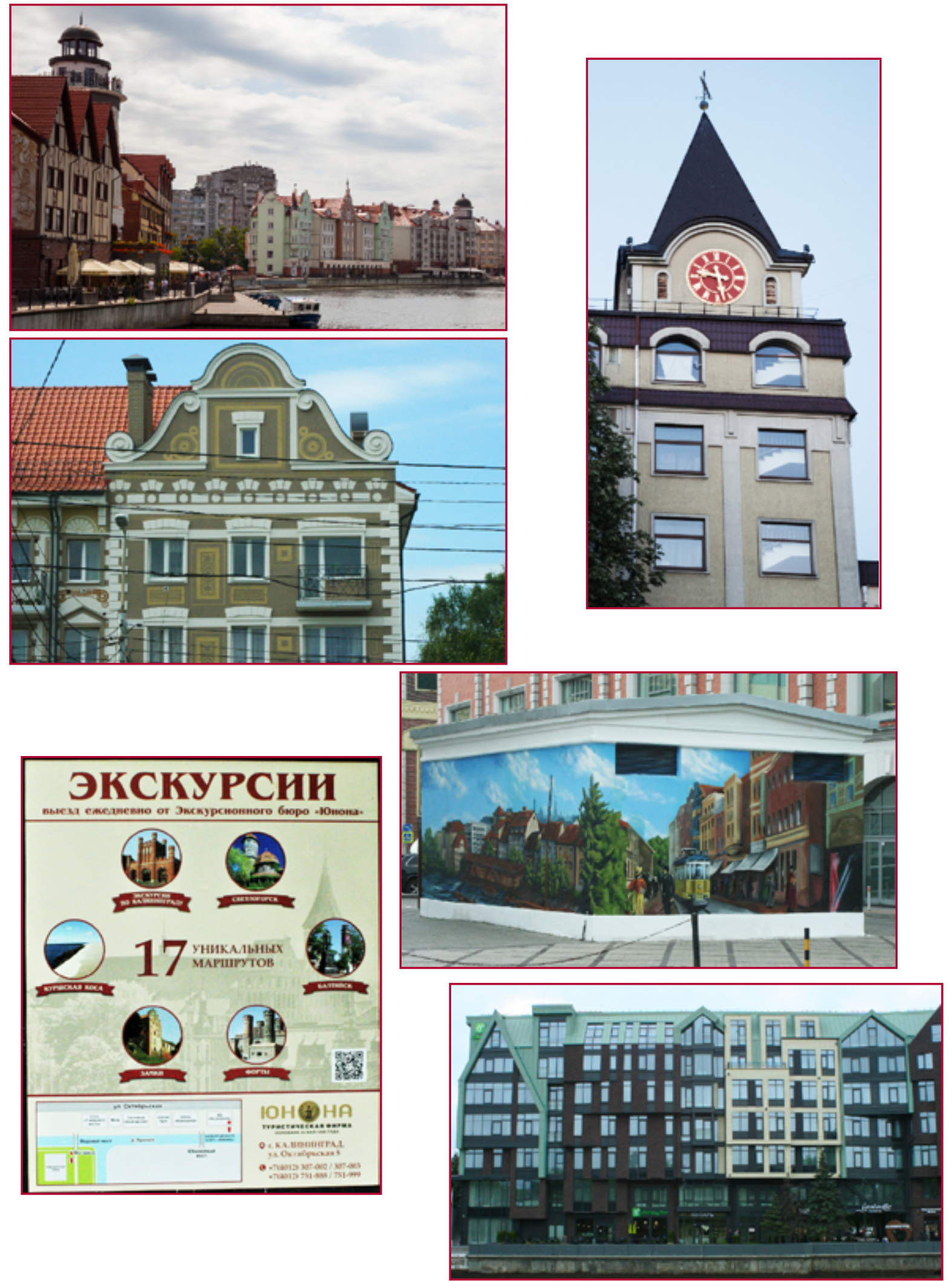

Figure 7. Planche photographique de l'identité allemande de la ville, rematérialisée en parc thématique « Königsberg » dès le début des années 1990 ; Kaliningrad, juillet 2018 et mai 2019 - (C) Colin Horenbeek 


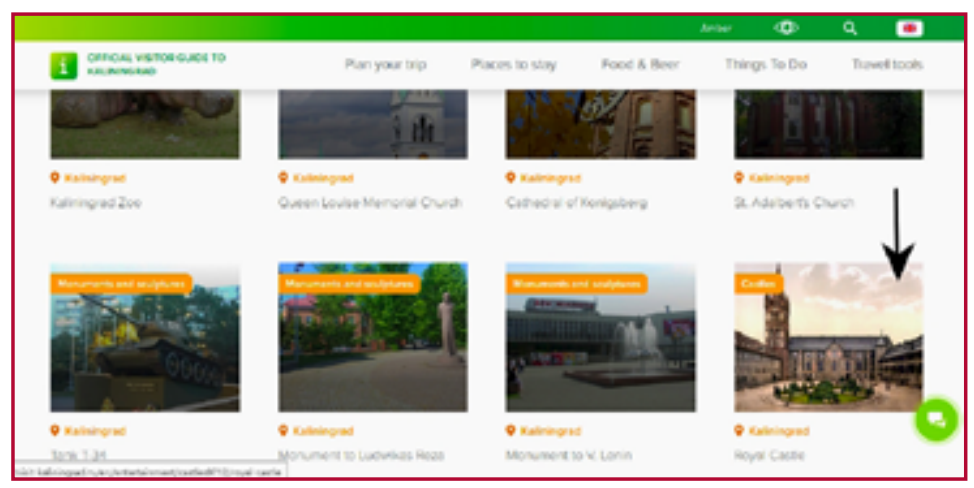

Figure 8. Capture d'écran du site de l'office du tourisme de Kaliningrad. Une photo d'archive recolorée suggère aux visiteurs que le Château royal de Königsberg existe toujours ; juillet 2019 - () Colin Horenbeek.

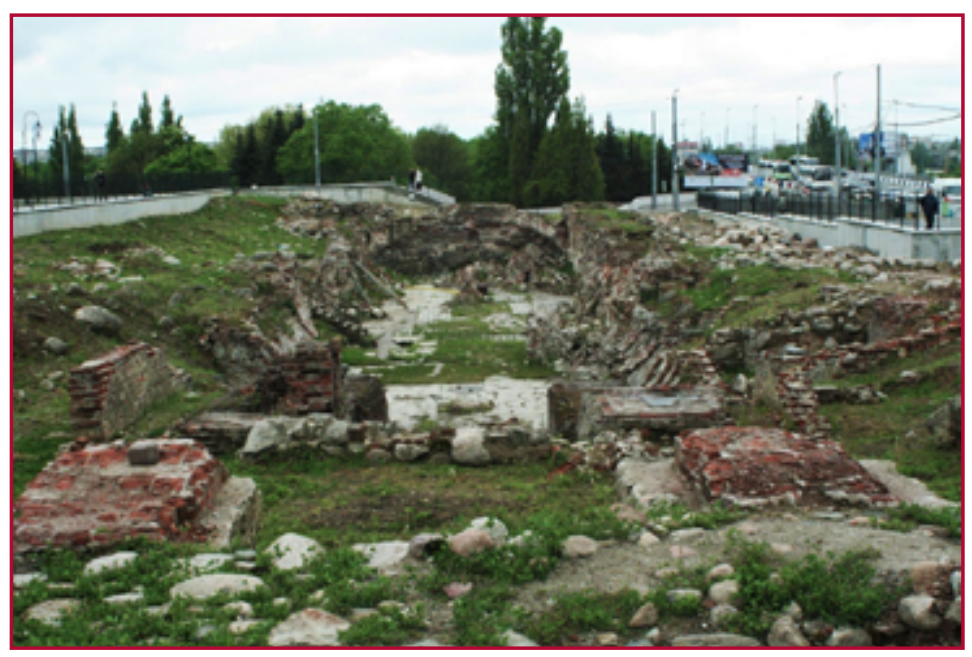

Figure 9. Ruines du Château de Königsberg ; Kaliningrad, mai 2019 - (C) Colin Horenbeek. 


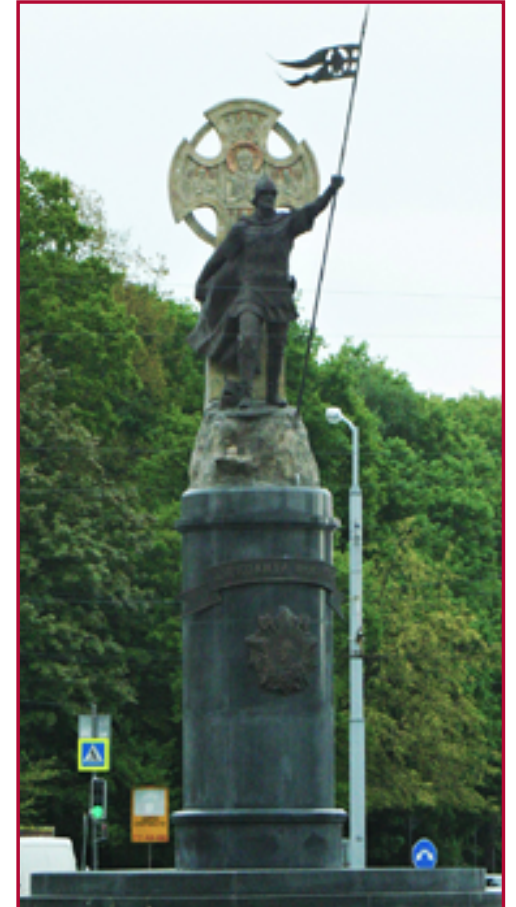

Statue d'Alexandre Nevski (2018)

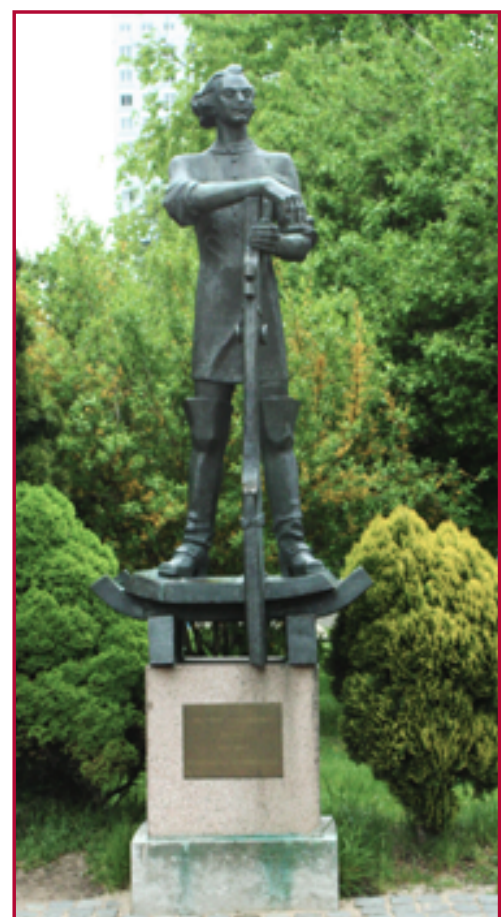

Statue du tsar Pierre $\mathrm{I}^{\mathrm{er}}(2017)$

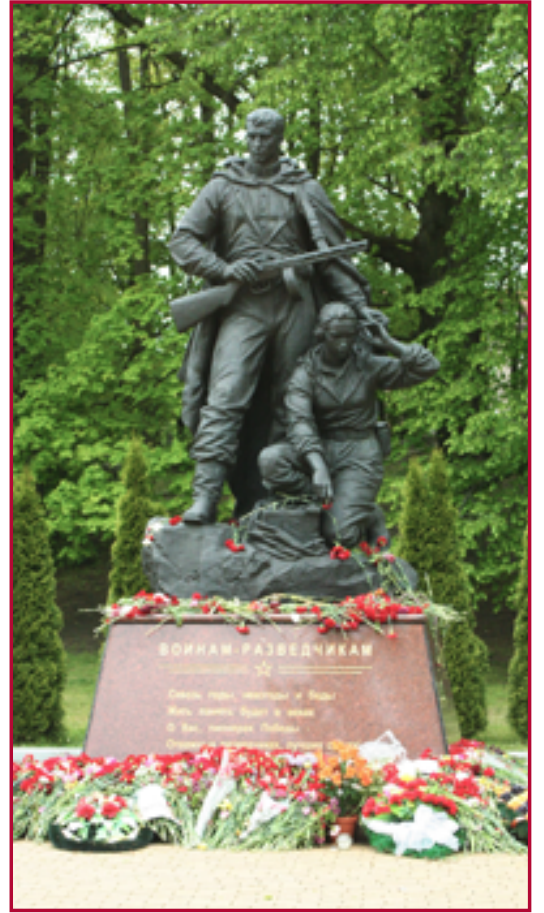

Statue en l'honneur des héros de la Seconde Guerre mondiale (2013)

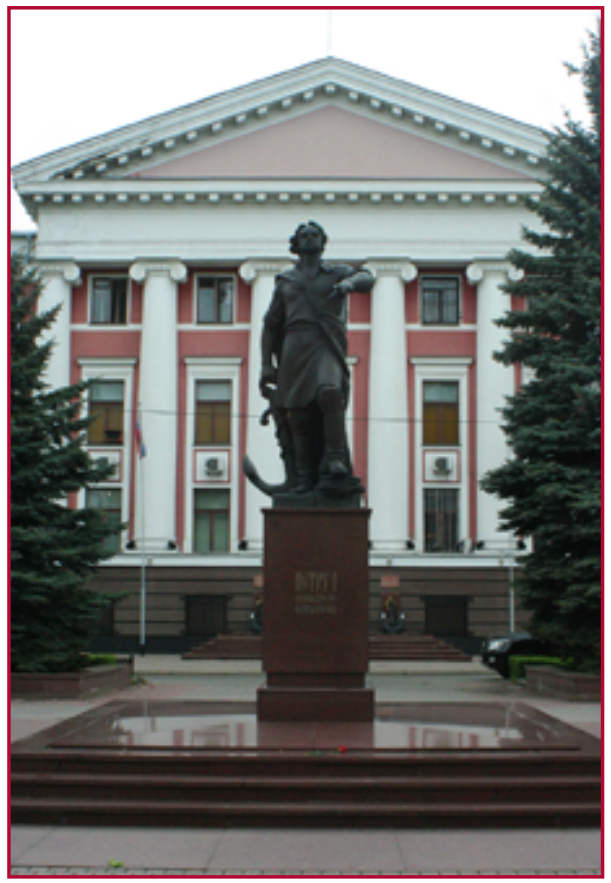

Statue du tsar Pierre I ${ }^{\text {er }}$ (2003)

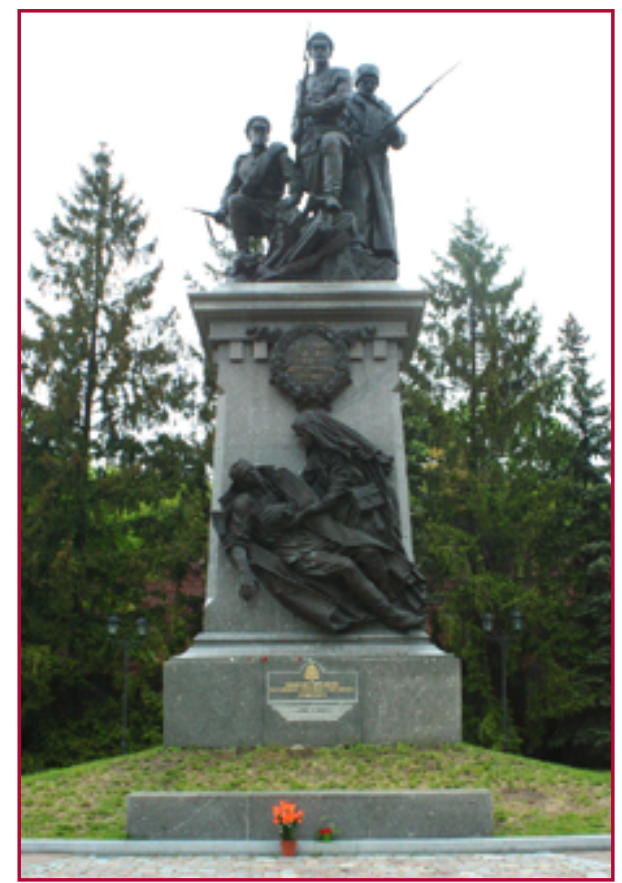

Statue en l'honneur des héros de la Première Guerre mondiale (2014)

Figure 10. Planche photographique illustrant la matérialisation de la nation : les " nouveaux " héros russes à Kaliningrad ; Kaliningrad, mai 2019 - (C) Colin Horenbeek 


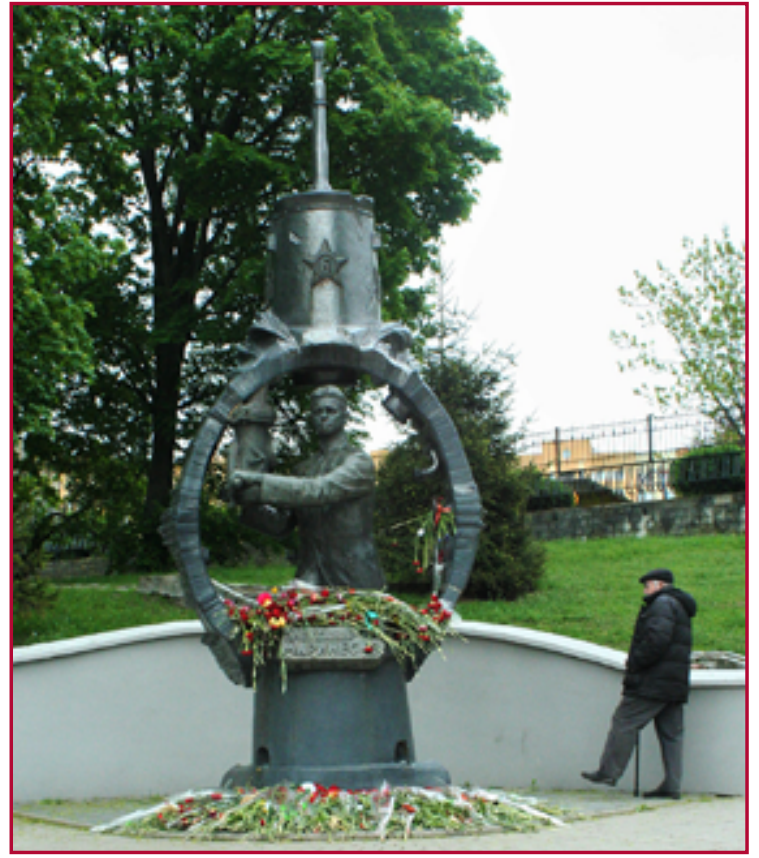

Statue d'Alexandre Marinesko (2011)

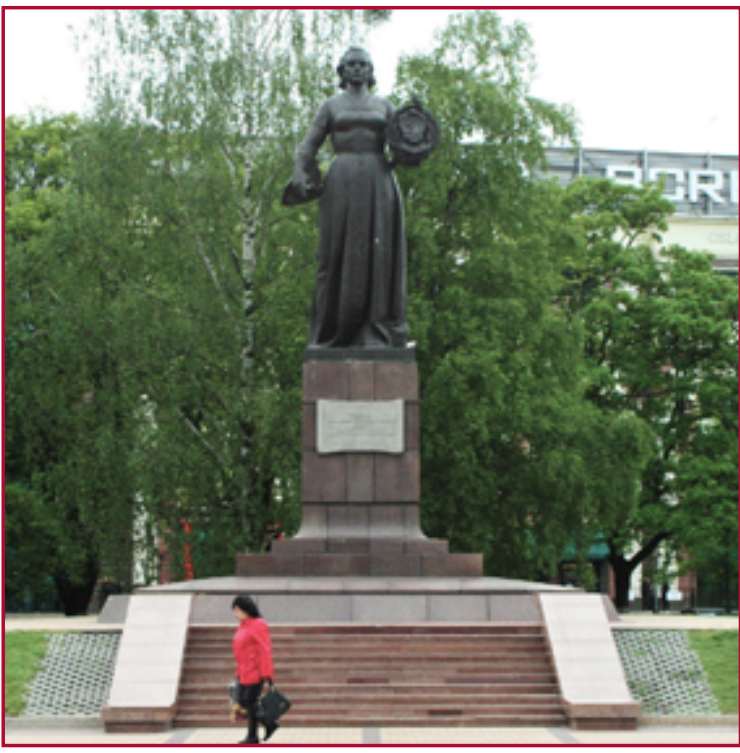

Statue en l'honneur de la « Mère Patrie »(1974), renommée en « Mère Russie » en 2018

Figure 10. Planche photographique illustrant la matérialisation de la nation : les « nouveaux » héros russes à Kaliningrad ; Kaliningrad, mai 2019 - (C) Colin Horenbeek 


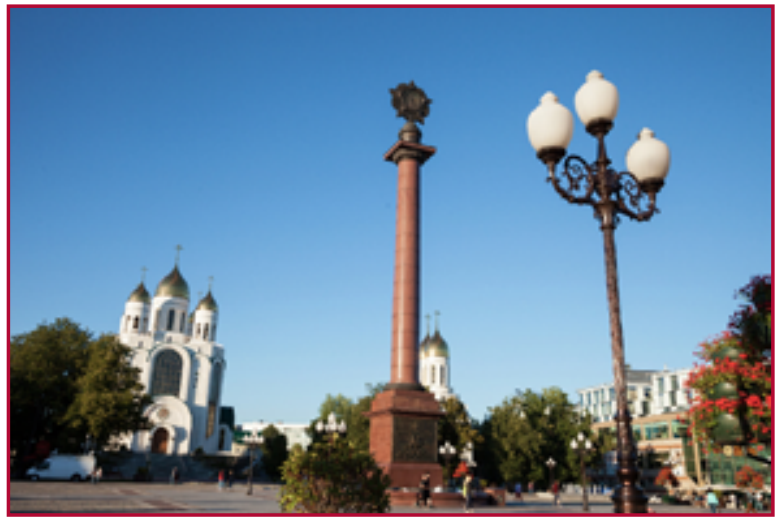

Figure 11. Cathédrale du Christ-Sauveur et Colonne de la Victoire, toutes deux construites en 2005, sur la place de la Victoire ; Kaliningrad, juillet 2018. c) Colin Horenbeek.

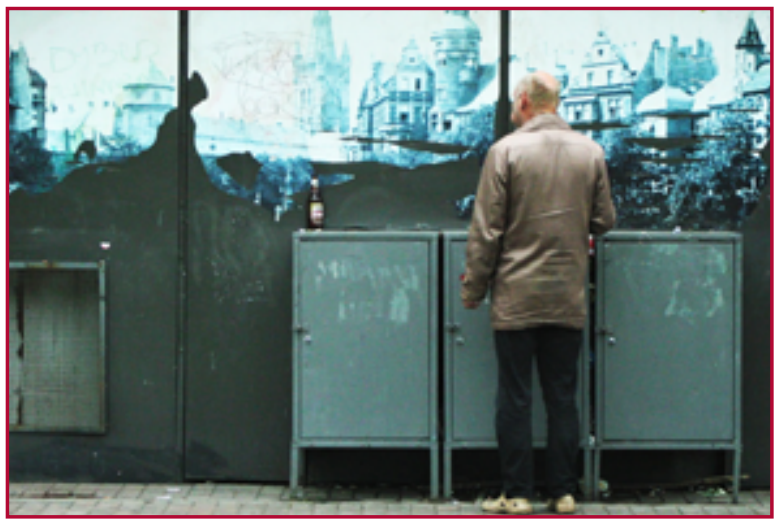

Figure 13. Un homme sans-abri derrière un kiosque. Il fait face à une représentation de Königsberg, délavée, déchirée, annotée, entre vision d'espoir et illusion perdue ; Kaliningrad, mai 2019.

(C) Colin Horenbeek.

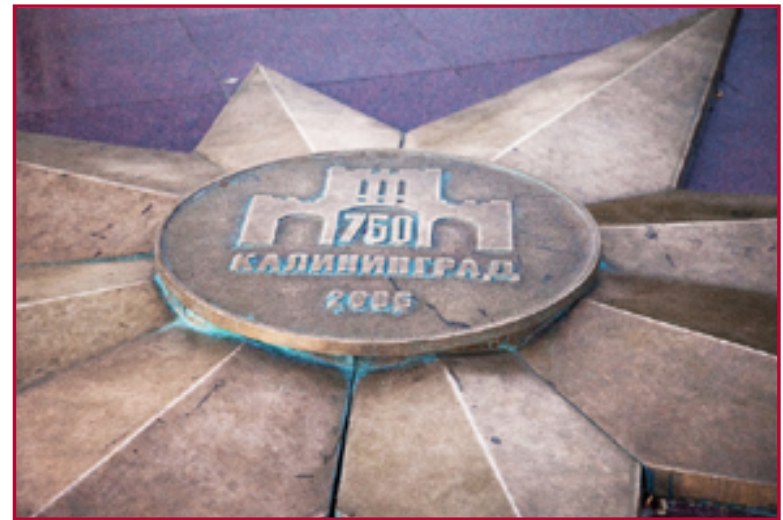

Figure 12. Stèle commémorant les « 750 ans » de Kaliningrad en 2005, sur la place de la Victoire ; Kaliningrad, juillet 2018.

(c) Colin Horenbeek.

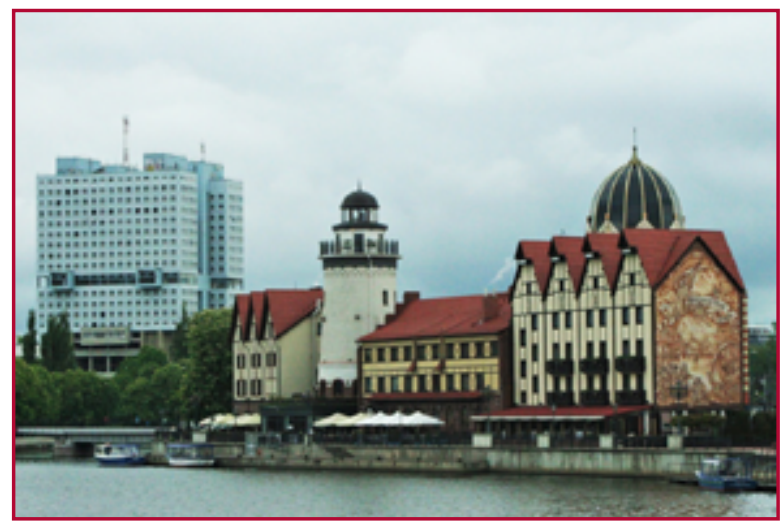

Figure 14. Le Soviet suprême et le Village des Pêcheurs, respectivement symboles des identités soviétique et allemande ; Kaliningrad, mai 2019. (c) Colin Horenbeek. 